Document downloaded from:

http://hdl.handle.net/10251/50602

This paper must be cited as:

Pedraza Aguilera, T.; Rodríguez López, J.; Romaguera Bonilla, S. (2014). Convergence of fuzzy sets with respect to the supremum metric. Fuzzy Sets and Systems. 245:83-100. doi:10.1016/j.fss.2014.03.005.

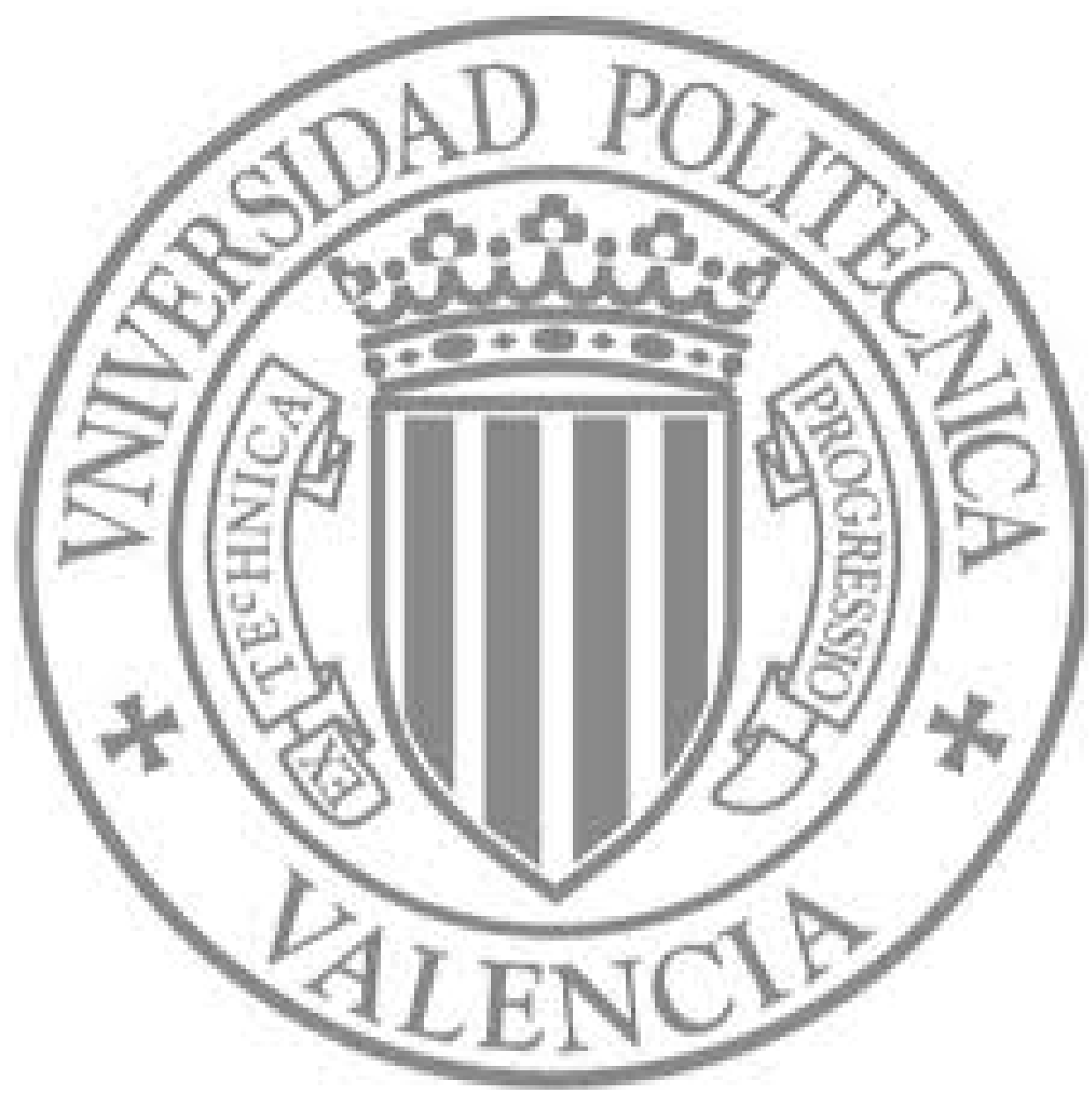

The final publication is available at

http://dx.doi.org/10.1016/j.fss.2014.03.005

Copyright Elsevier 


\title{
CONVERGENCE OF FUZZY SETS WITH RESPECT TO THE SUPREMUM METRIC
}

\author{
TATIANA PEDRAZA, JESÚS RODRÍGUEZ-LÓPEZ*,* AND SALVADOR ROMAGUERA*
}

\begin{abstract}
We characterize the convergence of fuzzy sets in the supremum metric given by the supremum of the Hausdorff distances of the $\alpha$-cuts of the fuzzy sets. We do it by dividing this metric into its lower and upper quasipseudometric parts. This characterization is given in the more general context with no assumption on the fuzzy sets. Furthermore, motivated from the theory of Convex Analysis, we also provide some results about the behaviour of the convergence in the supremum metric with respect to maximizers.
\end{abstract}

2010 Mathematics Subject Classification: 54A40, 54A20, 54B20, 54E35

Keywords and Phrases: supremum metric, fuzzy set, $\Gamma$-convergence, Hausdorff metric

\section{INTRODUCTION}

Throughout this paper we will use the letters $\mathbb{N}, \mathbb{R}$ and $I$ to denote the set of positive integer numbers, the set of real numbers and the unit interval, respectively. We also denote by $d_{e}$ the usual Euclidean metric on $\mathbb{R}$ or on any of its subsets. Furthermore, if $(X, d)$ is a metric space we denote by $B_{d}(x, \varepsilon)\left(\operatorname{resp} . \overline{B_{d}}(x, \varepsilon)\right)$ the open ball (resp. the closed ball) with center $x \in X$ and radius $\varepsilon>0$. We also write $B_{d}(A, \varepsilon)=\cup_{a \in A} B_{d}(a, \varepsilon)$ whenever $A$ is a nonempty subset of $X$.

The supremum metric has its origins in the theory of convergence of fuzzy sets $[10,12,13,14,15,16,17,18,22,23,26]$. Recall that a fuzzy set $f$ on a topological space $(X, \tau)$, as originally defined by Zadeh, is a function $f$ from $X$ to the closed unit interval $I$. The endograph or hypograph of $f$ is the set of all the points below its graph, i. e. end $f=\{(x, \alpha) \in X \times I: \alpha \leq f(x)\}$ whereas the epigraph are all the points over the graph, i. e. epi $f=\{(x, \alpha) \in X \times I: f(x) \leq \alpha\}$. The superlevel set of $f$ at height $\alpha$ (or $\alpha$-cut) is $[f]^{\alpha}=\{x \in X: \alpha \leq f(x)\}$ where $0<\alpha \leq 1$. The support of $f$ is $[f]^{0}=\overline{\cup_{0<\alpha \leq 1}[f]^{\alpha}}$ and its supported endograph or sendograph is the endograph where the first coordinate is restricted to the support of $f$, i. e. send $f=\operatorname{end} f \cap\left([f]^{0} \times I\right)$.

Furthermore, we recall that

- the upper limit of $f$ in $a \in X$ is given by

$$
\bar{f}(a)=\limsup _{x \rightarrow a} f(x)=\inf _{V \in \mathcal{N}(a)} \sup _{x \in V} f(x) ;
$$

\footnotetext{
Date: 9 January 2014.

* Corresponding author (e-mail: jrlopez@mat.upv.es, Tel.: +34963877663, Fax: $+34963877669)$

* The second and third authors thank the support of the Ministry of Economy and Competitiveness of Spain under grant MTM2012-37894-C02-01.
} 
- the lower limit of $f$ in $a \in X$ is given by

$$
\underline{f}(a)=\liminf _{x \rightarrow a} f(x)=\sup _{V \in \mathcal{N}(a)} \inf _{x \in V} f(x),
$$

where $\mathcal{N}(a)$ denotes the neighborhood filter of $a$.

Then a fuzzy set $f$ is upper semicontinuous (resp. lower semicontinuous) at $a$ if $f(a)=\limsup _{x \rightarrow a} f(x)$ (resp. $f(a)=\liminf _{x \rightarrow a} f(x)$ ), equivalently limsup $\sin _{x \rightarrow a} f(x) \leq$ $f(a)$ (resp. $f(a) \leq \liminf _{x \rightarrow a} f(x)$ ). We denote by $U S C(X)$ the family of all upper semicontinuous fuzzy sets on $X$. Furthermore, $f$ is continuous at $a$ if and only if $\limsup _{x \rightarrow a} f(x)=\liminf _{x \rightarrow a} f(x)$.

We also notice that $\overline{\operatorname{end} f}=\operatorname{end} \bar{f}$ (resp. $\overline{\operatorname{epi} f}=$ epif), where closure is taken in $\tau \times \tau\left(d_{e}\right)$, so $f$ is upper semicontinuous (resp. lower semicontinuous) if and only if end $f$ (resp. epif) is closed.

In the literature, we can find several convergences for fuzzy sets whose properties and relationships have been studied by several authors $[14,17,25]$. One of the most used is the convergence in the supremum metric. Recall that, given a metric space $(X, d)$, the supremum distance between two fuzzy sets $f, g$ on $X$ is defined as

$$
d_{\infty}(f, g)=\sup _{0<\alpha \leq 1} H_{d}\left([f]^{\alpha},[g]^{\alpha}\right)
$$

where $H_{d}$ is the Hausdorff extended pseudometric between subsets of $X$ given by

$$
H_{d}(A, B)=\max \left\{e_{d}(A, B), e_{d}(B, A)\right\}
$$

where

is the excess of $A$ over $B$.

$$
e_{d}(A, B)= \begin{cases}\sup _{a \in A} d(a, B) & \text { if } A \neq \varnothing \\ 0 & \text { if } A=\varnothing\end{cases}
$$

We also observe that

$$
H_{d}(A, B)=\max \left\{\inf \left\{\varepsilon>0: A \subseteq B_{d}(B, \varepsilon)\right\}, \inf \left\{\varepsilon>0: B \subseteq B_{d}(A, \varepsilon)\right\}\right\} .
$$

where we understand that the infimum is $+\infty$ if no such $\varepsilon$ exists.

The main purpose of this paper is to obtain a general characterization of $d_{\infty^{-}}$ convergence in $\mathcal{F}(X)$ (the family of all fuzzy sets over $X$, i. e. all the $[0,1]$ valued functions defined on $X)$. The antecedents of our approach may be found in [14], where the authors gave a characterization of the $d_{\infty}$-convergence (or $\mathcal{D}$ convergence) of upper semicontinuous fuzzy sets $f$ with nonempty compact support and $[f]^{1} \neq \varnothing$ (see Corollaries 3 and 6 ). Under these assumptions, several authors have studied the relationship between this convergence and the hypo-convergence (or $\Gamma$-convergence) [14], Hausdorff convergence of supported endographs (or $\mathcal{H}$ convergence) [20] and Hausdorff convergence of superlevel sets at a fixed height (also named $\mathcal{L}$-convergence or levelwise convergence) [17]. Nevertheless, we cannot find results which do not impose conditions on the fuzzy sets. To achieve our study, and as it is usual in hyperspace theory, we will split this metric in two halves: the lower supremum (extended) quasi-pseudometric and the upper supremum (extended) quasi-pseudometric. Then, we will study the relationship between the convergences associated to these two (extended) quasi-pseudometrics and some uniform convergences of certain functionals which will allow us to locate these convergences in the lattice of convergence of functions. Finally, we will characterize the aforementioned convergences by means of set-theoretic convergences. 
Furthermore, since $\Gamma$-convergence is tied to Convex Analysis $[2,3,4,5,6,8$, $21,24]$, we study some questions of convergence in the supremum metric from this point of view. Specifically, we analyze how this convergence behaves with respect to maximizers. For example, we prove that if $\left\{f_{\lambda}\right\}_{\lambda \in \Lambda}$ is a net of fuzzy sets converging to an upper semicontinuous fuzzy set $f$ and $\left\{x_{\lambda}\right\}_{\lambda \in \Lambda}$ is a net in $X$ converging to $x$, where $x_{\lambda}$ is a maximizer of $f_{\lambda}$ for all $\lambda \in \Lambda$, then $x$ is a maximizer of $f$.

\section{Convergences on $[0,1]$}

We will introduce some convergences on the unit interval $I=[0,1]$ of $\mathbb{R}$ which will be useful in our study. These convergences can also be considered in the general context of the set $\overline{\mathbb{R}}=\mathbb{R} \cup\{-\infty,+\infty\}$.

Definition 1. Let $\left\{x_{\lambda}\right\}_{\lambda \in \Lambda}$ be a net in I. Then

- the lower limit of $\left\{x_{\lambda}\right\}_{\lambda \in \Lambda}$ is

$$
\liminf _{\lambda \in \Lambda} x_{\lambda}=\sup _{\lambda^{\prime} \in \Lambda} \inf _{\lambda \geq \lambda^{\prime}} x_{\lambda}
$$

- the upper limit of $\left\{x_{\lambda}\right\}_{\lambda \in \Lambda}$ is

$$
\limsup _{\lambda \in \Lambda} x_{\lambda}=\inf _{\lambda^{\prime} \in \Lambda} \sup _{\lambda \geq \lambda^{\prime}} x_{\lambda} .
$$

Notice that $\lim _{\lambda \in \Lambda} x_{\lambda}=x$ in the usual topology of $I$ if and only if lim $\sup _{\lambda \in \Lambda} x_{\lambda}=$ $\liminf _{\lambda \in \Lambda} x_{\lambda}=x$.

From this, we consider the following topological convergences on $I$.

Definition 2. A net $\left\{x_{\lambda}\right\}_{\lambda \in \Lambda}$ in I is said to be

- lower convergent to $x \in I$, and we write $x \in \lim _{\lambda \in \Lambda}^{\mathcal{L}} x_{\lambda}$, if $x \leq \liminf _{\lambda \in \Lambda} x_{\lambda}$;

- upper convergent to $x \in I$, and we write $x \in \lim _{\lambda \in \Lambda}^{\mathcal{U}} x_{\lambda}$, if $\lim \sup _{\lambda \in \Lambda} x_{\lambda} \leq$ $x$.

Consequently,

$$
\liminf _{\lambda \in \Lambda} x_{\lambda}=\max \lim _{\lambda \in \Lambda} \mathcal{L}^{\mathcal{L}} x_{\lambda} \text { and } \limsup _{\lambda \in \Lambda} x_{\lambda}=\min \lim _{\lambda \in \Lambda}{ }^{u} x_{\lambda} .
$$

Remark 1. It is easy to check that lower convergence (resp. upper convergence) is convergence in the topology of the lower quasi-pseudometric $\ell$ (resp. upper quasipseudometric $u)$ given by $\ell(x, y)=\max \{x-y, 0\} \quad$ (resp. $u(x, y)=\ell(y, x)$ ) for all $x, y \in I$. Furthermore, the lower convergence (resp. upper convergence) is compatible with the lower topology (resp. upper topology) on I whose open sets are of the form $(a, 1]$ (resp. [0,a)) where $a \in I$.

In the theory of continuous lattices, the lower topology on I is known as the Scott topology [11] and its open sets are characterized by the following: $O$ is Scott-open if and only if $O$ is an upper set and if $\sup D \in O$ then $D \cap O \neq \varnothing$ for every directed set $D$.

Remark 2. We observe that a fuzzy set $f$ on a metric space $(X, d)$ is upper semicontinuous (resp. lower semicontinuous) at $x \in X$ if and only if $f$ is continuous at $x$ when $I$ is endowed with the upper (resp. lower) quasi-pseudometric. Consequently, $f$ is upper semicontinuous (resp. lower semicontinuous) at $x$ if for every $\varepsilon>0$ we can find $\delta_{\varepsilon}>0$ such that $f(y)-f(x)<\varepsilon$ (resp. $\left.f(x)-f(y)<\varepsilon\right)$ for all $y \in B_{d}\left(x, \delta_{\varepsilon}\right)$. 
Then we say that a fuzzy set $f$ is uniformly upper semicontinuous if for every $\varepsilon>0$ we can find $\delta_{\varepsilon}>0$ such that

$$
f(y)-f(x)<\varepsilon
$$

whenever $d(x, y)<\delta_{\varepsilon}$.

We denote by UUSC $(X)$ the family of all uniformly upper semicontinuous fuzzy sets on $X$.

We introduce now two other convergences.

Definition 3. Given $x \in I$, we say that a net $\left\{x_{\lambda}\right\}_{\lambda \in \Lambda}$ in $I$ is:

- $\mathcal{S}_{\leq}$-convergent to $x$ if there exists $\lambda_{0} \in \Lambda$ such that $x \leq x_{\lambda}$ for all $\lambda \geq \lambda_{0}$;

- $\mathcal{S}_{\geq}$-convergent to $x$ if there exists $\lambda_{0} \in \Lambda$ such that $x \geq x_{\lambda}$ for all $\lambda \geq \lambda_{0}$.

Remark 3. We observe that $\mathcal{S}_{\leq}$-convergence coincides with the convergence in the Alexandroff topology associated with the partial order $\leq$ of $I$, whose open sets constitute the topology

$\tau_{\leq}=\{O \subseteq I:$ if $x, y \in I, x \in O$ and $x \leq y \Rightarrow y \in O\}=\{[a, 1]: a \in I\} \cup\{\varnothing\}$.

We also notice that if $A \subseteq I$ then the closure of $A$ in $\tau_{\leq}$is $\bar{A}=\downarrow A=\{x \in I$ : $x \leq a$ for some $a \in A\}$.

In a similar way, $\mathcal{S}_{\geq}$-convergence coincides with the convergence in the Alexandroff topology associated with the partial order $\geq$ of $I$, whose open sets are

$\tau_{\geq}=\{O \subseteq I:$ if $x, y \in I, x \in O$ and $x \geq y \Rightarrow y \in O\}=\{[0, a]: a \in I\} \cup\{\varnothing\}$.

Furthermore (compare with equation (2))

$$
\liminf _{\lambda \in \Lambda} x_{\lambda}=\sup \lim _{\lambda \in \Lambda}{ }^{\tau} \leq x_{\lambda} \text { and } \limsup _{\lambda \in \Lambda} x_{\lambda}=\inf \lim _{\lambda \in \Lambda} \tau^{\tau} x_{\lambda},
$$

and, in general, $\left\{x_{\lambda}\right\}_{\lambda \in \Lambda}$ is not $\tau_{\leq}$-convergent (resp. $\tau_{\geq}$-convergent) to $\lim \inf _{\lambda \in \Lambda} x_{\lambda}$ (resp. $\lim \sup _{\lambda \in \Lambda} x_{\lambda}$ ). Therefore, the Alexandroff topology $\tau_{\leq}\left(\right.$resp. $\tau_{\geq}$) is strictly finer than the lower topology (resp. upper topology).

We introduce now other two convergences which will be useful later on.

Definition 4. Given $x \in I$, we say that a net $\left\{x_{\lambda}\right\}_{\lambda \in \Lambda}$ in $I$ is:

- $\mathcal{S}_{<}$-convergent to $x$ if there exists $\lambda_{0} \in \Lambda$ such that $x<x_{\lambda}$ for all $\lambda \geq \lambda_{0}$;

- $\mathcal{S}_{>}$-convergent to $x$ if there exists $\lambda_{0} \in \Lambda$ such that $x>x_{\lambda}$ for all $\lambda \geq \lambda_{0}$.

Remark 4. Notice that the two above convergences are not topological, $i$. e. there does not exist any topology whose associated convergence is equivalent to one of the above convergences. This is due to the fact that constant sequences are not convergent in these convergences. Nevertheless, they satisfy the rest of the axioms for being a topological convergence (see [19, page 64]).

Furthermore, the finest topology whose convergence is coarser than $\mathcal{S}_{<}$-convergence (resp. $\mathcal{S}_{>}$-convergence) is the Alexandroff topology $\tau_{\leq}$(resp. $\left.\tau_{\geq}\right)$.

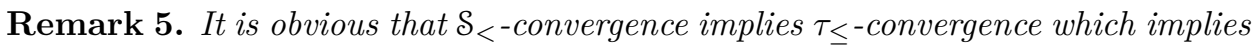
lower convergence. In the same way, $\mathcal{S}_{>}$-convergence implies $\tau_{\geq}$-convergence which implies upper convergence.

\section{Convergences of FuZzy Sets}

By $\mathcal{F}(X)$ we denote the family of all fuzzy sets over $X$, i. e. all the $[0,1]$-valued functions defined over $X$. 


\subsection{Pointwise convergence.}

Definition 5 ([1, Page 71$])$. Let $(X, \tau)$ be a topological space and $\left\{f_{\lambda}\right\}_{\lambda \in \Lambda}$ a net in $\mathcal{F}(X)$, where $I$ is endowed with a convergence $\mathcal{S}$. We say that $\left\{f_{\lambda}\right\}_{\lambda \in \Lambda}$ is $\mathcal{S}$ pointwise convergent to $f$ at $x \in X$ if the sequence $\left\{f_{\lambda}(x)\right\}_{\lambda \in \Lambda}$ is $\mathcal{S}$-convergent to $f(x)$.

If $\left\{f_{\lambda}\right\}_{\lambda \in \Lambda}$ is $\mathcal{S}$-pointwise convergent to $f$ at every $x \in X$, we just say that $\left\{f_{\lambda}\right\}_{\lambda \in \Lambda}$ is $\mathcal{S}$-pointwise convergent to $f$.

When we particularize the above definition for the lower and upper convergences we have the following:

Definition 6. Let $(X, \tau)$ be a topological space. A net $\left\{f_{\lambda}\right\}_{\lambda \in \Lambda}$ in $\mathcal{F}(X)$ is said to be:

- lower pointwise convergent to $f$ at $x \in X$ if $\liminf _{\lambda \in \Lambda} f_{\lambda}(x) \geq f(x)$;

- upper pointwise convergent to $f$ at $x \in X$ if $\limsup _{\lambda \in \Lambda} f_{\lambda}(x) \leq f(x)$;

- pointwise convergent to $f$ at $x \in X$ if it is both lower and upper pointwise convergent to $f$ at $x, i$. e. $\lim _{\lambda \in \Lambda} f_{\lambda}(x)=f(x)$.

When the above conditions are satisfied for all $x \in X$ we just say that $\left\{f_{\lambda}\right\}_{\lambda \in \Lambda}$ is lower pointwise convergent (resp. upper pointwise convergent, pointwise convergent) to $f$.

Remark 6. Notice that $\left\{f_{\lambda}\right\}_{\lambda \in \Lambda}$ is lower pointwise convergent to $f$ if and only if end $f \subseteq$ end li $f_{\lambda}$ where $\left(\operatorname{li} f_{\lambda}\right)(x)=\liminf _{\lambda \in \Lambda} f_{\lambda}(x)$ for all $x \in X$.

In a similar way, $\left\{f_{\lambda}\right\}_{\lambda \in \Lambda}$ is upper pointwise convergent to $f$ if and only if end ls $f_{\lambda} \subseteq$ end $f$ where $\left(\operatorname{ls} f_{\lambda}\right)(x)=\lim \sup _{\lambda \in \Lambda} f_{\lambda}(x)$.

Consequently, $\left\{f_{\lambda}\right\}_{\lambda \in \Lambda}$ is pointwise convergent to $f$ if and only if end ls $f_{\lambda}=$ end li $f_{\lambda}=$ end $f$. This statement is also valid considering epigraphs instead of endographs.

If we consider in Definition 5 the $\tau_{\leq}$-convergence and $\tau_{\geq}$-convergence we have the following:

Definition 7. Let $(X, \tau)$ be a topological space. A net $\left\{f_{\lambda}\right\}_{\lambda \in \Lambda}$ in $\mathcal{F}(X)$ is said to be:

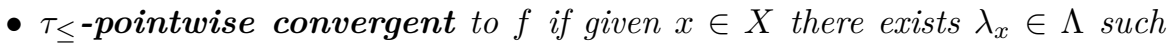
that $f(x) \leq f_{\lambda}(x)$ for all $\lambda \geq \lambda_{x}$;

- $\tau_{\geq}$-pointwise convergent to $f$ if given $x \in X$ there exists $\lambda_{x} \in \Lambda$ such that $f(x) \geq f_{\lambda}(x)$ for all $\lambda \geq \lambda_{x}$.

If the above inequalities are strict we say that $\left\{f_{\lambda}\right\}_{\lambda \in \Lambda}$ is $\mathcal{S}_{<- \text {pointwise convergent }}$ (resp. $\mathcal{S}_{>}$-pointwise convergent) to $f$.

3.2. $\Gamma$-convergence. The origins of the $\Gamma$-convergence, and its counterpart epiconvergence, are due to Wijsman [27] when he introduced the so-called infimal convergence for convex functions. The relationship between this convergence and Kuratowski-Painlevé convergence of epigraphs was explicitly observed by Klee in the review of Wijsman's paper, although it is implicit in the results of the paper.

Later on, $\Gamma$-convergence appeared again in nonconvex problems of minimization by De Giorgi and Franzoni [7].

Recall that if $(X, \tau)$ is a topological space and $\left\{A_{\lambda}\right\}_{\lambda \in \Lambda}$ is a net of subsets of $X$ then 
- the lower limit of $\left\{A_{\lambda}\right\}_{\lambda \in \Lambda}$ is the set

$$
\operatorname{Li} A_{\lambda}=\left\{x \in X: A_{\lambda} \cap V \neq \varnothing \text { residually, for all } V \in \mathcal{N}(x)\right\} ;
$$

- the upper limit of $\left\{A_{\lambda}\right\}_{\lambda \in \Lambda}$ is the set

$$
\mathrm{Ls}_{\lambda}=\left\{x \in X: A_{\lambda} \cap V \neq \varnothing \text { cofinally, for all } V \in \mathcal{N}(x)\right\} .
$$

In this way, a net $\left\{A_{\lambda}\right\}_{\lambda \in \Lambda}$ of subsets of $X$ is lower Kuratowski-Painlevé convergent (resp. upper Kuratowski-Painlevé convergent; Kuratowski-Painlevé convergent) to $A[2]$ if $A \subseteq \operatorname{Li} A_{\lambda}$ (resp. $\operatorname{Ls} A_{\lambda} \subseteq A ; A=\operatorname{Li} A_{\lambda}=\operatorname{Ls} A_{\lambda}$ ). We also observe (see [2, Proposition 5.2.5]) that lower Kuratowski-Painlevé convergence is convergence in the lower Vietoris topology so it is always topological.

We recall that $\Gamma$-convergence is Kuratowski-Painlevé convergence of endographs of functions. Consequently, a net $\left\{f_{\lambda}\right\}_{\lambda \in \Lambda}$ in $\mathcal{F}(X)$ is said to be $\Gamma^{-}$-convergent to the fuzzy set $f$ if end $f \subseteq \mathrm{Li}$ end $f_{\lambda}$. It can be proved (see [2, Lemmas 5.3.3 and 5.3.4],[9, Proposition 1.9] for the epi-convergence version) that Li end $f_{\lambda}$ is the endograph of the fuzzy set given by

$$
\left(\operatorname{Li} f_{\lambda}\right)(x)=\inf _{V \in \mathcal{N}(x)} \liminf _{\lambda \in \Lambda} M_{f_{\lambda}}(V),
$$

where $M_{f_{\lambda}}(V)=\sup _{v \in V} f_{\lambda}(v)$.

Furthermore, the net $\left\{f_{\lambda}\right\}_{\lambda \in \Lambda}$ is $\Gamma^{+}$-convergent to $f$ if Ls end $f_{\lambda} \subseteq$ end $f$. The upper limit of the endographs of the elements of the net $\left\{f_{\lambda}\right\}_{\lambda \in \Lambda}$ is the endograph of the fuzzy set

$$
\left(\operatorname{Ls} f_{\lambda}\right)(x)=\inf _{V \in \mathcal{N}(x)} \limsup _{\lambda \in \Lambda} M_{f_{\lambda}}(V) .
$$

Remark 7. We notice that if $(X, d)$ is a metric space and $\left\{f_{\lambda}\right\}_{\lambda \in \Lambda}$ is a net in $\mathcal{F}(X)$ then

$$
\begin{aligned}
& \left(\operatorname{Li} f_{\lambda}\right)(x)=\inf _{\varepsilon>0} \liminf _{\lambda \in \Lambda} M_{f_{\lambda}}\left(B_{d}(x, \varepsilon)\right), \\
& \left(\operatorname{Ls} f_{\lambda}\right)(x)=\inf _{\varepsilon>0} \limsup _{\lambda \in \Lambda} M_{f_{\lambda}}\left(B_{d}(x, \varepsilon)\right) .
\end{aligned}
$$

It is not difficult to prove that if $\left\{f_{n}\right\}_{n \in \mathbb{N}}$ is a sequence of fuzzy sets in $\mathcal{F}(X)$ then

$$
\begin{aligned}
& \left(\operatorname{Li} f_{n}\right)(x)=\sup _{\left\{x_{n}\right\}_{n \in \mathbb{N}} \rightarrow x} \liminf _{n \rightarrow+\infty} f_{n}\left(x_{n}\right), \\
& \left(\operatorname{Ls} f_{n}\right)(x)=\sup _{\left\{x_{n}\right\}_{n \in \mathbb{N}} \rightarrow x} \limsup _{n \rightarrow+\infty} f_{n}\left(x_{n}\right) .
\end{aligned}
$$

We observe that, in contraposition with $\Gamma^{-}$-convergence, $\Gamma^{+}$-convergence is not topological in general. In fact, the sequence of fuzzy sets $\left\{f_{n}\right\}_{n \in \mathbb{N}}$ over $I$ endowed with the Euclidean topology whose elements are equal to the characteristic function $f$ of the set $] 1 / 2,1]$ is not $\Gamma^{+}$-convergent to $f$ since $\inf _{\varepsilon>0} \lim \sup _{n \in \mathbb{N}} M_{f_{n}}\left(B_{d}(1 / 2, \varepsilon)\right)=$ $1>f(1 / 2)=0$. The sequence $\left\{f_{n}\right\}_{n \in \mathbb{N}}$ is $\Gamma^{+}$-convergent to every fuzzy set greater than or equal to the characteristic function of the closed interval $[1 / 2,1]$.

Remark 8. Let $\left\{f_{\lambda}\right\}_{\lambda \in \Lambda}$ be a net of fuzzy sets defined on a topological space. We observe that:

end li $f_{\lambda} \subseteq \operatorname{Li}$ end $f_{\lambda}=$ end $\operatorname{Li} f_{\lambda} \quad$ and $\quad$ end $\operatorname{ls} f_{\lambda} \subseteq \operatorname{Ls}$ end $f_{\lambda}=$ end $\operatorname{Ls} f_{\lambda}$.

Suppose that $(x, \alpha) \in$ end li $f_{\lambda}$, i. e. $\alpha \leq \liminf _{\lambda \in \Lambda} f_{\lambda}(x)$. Let $\varepsilon>0$. Then $\alpha-\varepsilon / 2<\inf _{\lambda \geq \lambda^{\prime}} f_{\lambda}(x)$ for all $\lambda^{\prime}$ greater than a certain $\lambda_{\varepsilon}$. Consequently, $(x, \alpha-$ 
$\varepsilon / 2) \in$ end $f_{\lambda} \cap(V \times(\alpha-\varepsilon, \alpha+\varepsilon))$ for all $\lambda \geq \lambda_{\varepsilon}$ and all neighborhood $V$ of $x$. Therefore, $(x, \alpha) \in \operatorname{Li}$ end $f_{\lambda}$.

To prove the other inclusion we can proceed in a similar way but taking into account that if $\alpha \leq \lim \sup _{\lambda} f_{\lambda}(x)$ then for each $\lambda \in \Lambda$ we can find $\lambda^{\prime} \geq \lambda$ such that $\alpha-\varepsilon / 2<f_{\lambda^{\prime}}(x)$.

For first countable spaces, $\Gamma$-convergence admits a very useful characterization.

Theorem 1 (cf. [2, Theorem 5.3.5], [21, Propositions 8.6.1 and 8.6.2], [24, Proposition 7.2]). Given a first countable topological space $(X, \tau)$, a sequence $\left\{f_{n}\right\}_{n \in \mathbb{N}}$ in $\mathcal{F}(X)$ is

- $\Gamma^{-}$convergent to $f$ if and only if for every $x \in X$ there exists a sequence $\left\{x_{n}\right\}_{n \in \mathbb{N}}$ convergent to $x$ such that $f(x) \leq \liminf _{n \rightarrow+\infty} f_{n}\left(x_{n}\right)$;

- $\Gamma^{+}$-convergent to $f$ if and only if $\lim _{\sup _{n \rightarrow+\infty}} f_{n}\left(x_{n}\right) \leq f(x)$ for every sequence $\left\{x_{n}\right\}_{n \in \mathbb{N}}$ convergent to $x$.

Although in general there is no relationship between $\Gamma$-convergence and pointwise convergence [2, p. 156], [9, Theorem 2.18], by the above remark we can obtain the following:

Proposition 1. Let $(X, \tau)$ be a topological space and let $\left\{f_{\lambda}\right\}_{\lambda \in \Lambda}$ be a net in $\mathcal{F}(X)$. Then:

(1) if $\left\{f_{\lambda}\right\}_{\lambda \in \Lambda}$ is lower pointwise convergent to $f$ then it is $\Gamma^{-}$-convergent to $f ;$

(2) if $\left\{f_{\lambda}\right\}_{\lambda \in \Lambda}$ is $\Gamma^{+}$-convergent to $f$ then it is upper pointwise convergent to $f$

(3) the set of limit points of the net $\left\{f_{\lambda}\right\}_{\lambda \in \Lambda}$ in the lower pointwise convergence and in the $\Gamma^{-}$-convergence coincide if and only if $\left(\operatorname{li} f_{\lambda}\right)(x)=\left(\operatorname{Li} f_{\lambda}\right)(x)$ for all $x \in X$;

(4) the set of limit points of the net $\left\{f_{\lambda}\right\}_{\lambda \in \Lambda}$ in the upper pointwise convergence and in the $\Gamma^{+}$-convergence coincide if and only if $\left(\operatorname{ls} f_{\lambda}\right)(x)=\left(\operatorname{Ls} f_{\lambda}\right)(x)$ for all $x \in X$;

Proof. The proof follows from Remarks 6 and 8.

The next example shows limiting counterexamples for the preceding result.

Example 1. Let us consider the sequence $\left\{f_{n}\right\}_{n \in \mathbb{N}}$ of spike functions on I given by

$$
f_{n}(x)= \begin{cases}2 n x & \text { if } 0 \leq x<\frac{1}{2 n} \\ 2-2 n x & \text { if } \frac{1}{2 n} \leq x<\frac{1}{n} \\ 0 & \text { if } \frac{1}{n} \leq x \leq 1\end{cases}
$$

If we endow I with the Euclidean metric, then it is clear that $\left\{f_{n}\right\}_{n \in \mathbb{N}}$ is pointwise convergent to the zero fuzzy set on $I$, so (li $\left.f_{n}\right)(x)=\liminf _{n \rightarrow+\infty} f_{n}(x)=0$ for all $x \in I$. Nevertheless,

$$
\left(\operatorname{Li} f_{n}\right)(x)=\left(\operatorname{Ls} f_{n}\right)(x)= \begin{cases}0 & \text { if } 0<x \leq 1 \\ 1 & \text { if } x=0\end{cases}
$$

Consequently, Li end $f_{n}=$ Ls end $\left.\left.f_{n}=(\{0\} \times I) \cup(] 0,1\right] \times\{0\}\right)$. From this, we can deduce that the sequence $\left\{f_{n}\right\}_{n \in \mathbb{N}}$ is $\Gamma^{-}$-convergent to the characteristic function of the singleton $\{0\}$ but it is not lower pointwise convergent to this fuzzy set. Furthermore, the sequence is upper pointwise convergent to the zero fuzzy set but it is not $\Gamma^{+}$-convergent to that fuzzy set. 
3.3. Uniform convergence. Recall that a net $\left\{f_{\lambda}\right\}_{\lambda \in \Lambda}$ in $\mathcal{F}(X)$ is uniform convergent to a fuzzy set $f$ if for each $\varepsilon>0$ there exists $\lambda_{\varepsilon} \in \Lambda$ such that for all $x \in X$

$$
\left|f(x)-f_{\lambda}(x)\right|<\varepsilon \text { for all } \lambda \geq \lambda_{\varepsilon} .
$$

Definition 6 splits the pointwise convergence in two convergences. The same can be made with the uniform convergence.

Definition 8. Let $(X, \tau)$ be a topological space and let $f$ be a fuzzy set on $X$. A net $\left\{f_{\lambda}\right\}_{\lambda \in \Lambda}$ in $\mathcal{F}(X)$ is:

- lower uniformly convergent to $f$ if for each $\varepsilon>0$ there exists $\lambda_{\varepsilon} \in \Lambda$ such that for all $x \in X$

$$
f(x)-f_{\lambda}(x)<\varepsilon \text { for all } \lambda \geq \lambda_{\varepsilon}
$$

- upper uniformly convergent to $f$ if for each $\varepsilon>0$ there exists $\lambda_{\varepsilon} \in \lambda$ such that for all $x \in X$

$$
f_{\lambda}(x)-f(x)<\varepsilon \text { for all } \lambda \geq \lambda_{\varepsilon} .
$$

From this it is clear that $\left\{f_{\lambda}\right\}_{\lambda \in \Lambda}$ is uniformly convergent to $f$ if it is both lower and upper uniformly convergent to $f$.

We next introduce other uniform convergences related to some convergences introduced in Section 2.

Definition 9. Let $(X, \tau)$ be a topological space and let $f$ be a fuzzy set on $X$. A net $\left\{f_{\lambda}\right\}_{\lambda \in \Lambda}$ in $\mathcal{F}(X)$ is:

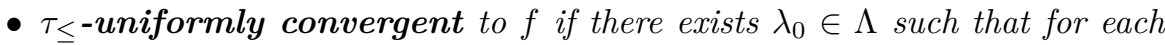
$x \in X$

$$
f(x) \leq f_{\lambda}(x) \text { for all } \lambda \geq \lambda_{0}
$$

- $\tau_{\geq}$-uniformly convergent to $f$ there exists $\lambda_{0} \in \Lambda$ such that for each $x \in X$

$$
f_{\lambda}(x) \leq f(x) \text { for all } \lambda \geq \lambda_{0} .
$$

If the above inequalities are strict we say that $\left\{f_{\lambda}\right\}_{\lambda \in \Lambda}$ is $\mathcal{S}_{<}$-uniformly convergent (resp. $\mathcal{S}_{>}$-uniformly convergent) to $f$.

Observe that simultaneous $\tau_{\leq}$-uniform convergence and $\tau_{\geq}$-uniform convergence of a net $\left\{f_{\lambda}\right\}_{\lambda \in \Lambda}$ to a function $f$ obviously implies that $f=f_{\lambda}$ residually. Furthermore, it is clear that $\tau_{\leq}$-uniform convergence (resp. $\tau_{\geq}$-uniform convergence) implies lower uniform convergence (resp. upper uniform convergence). It is easy to construct examples to check that the converse is not true in general.

\section{The SUPREMUM METRIC}

Let $(X, d)$ be a metric space. The supremum metric $d_{\infty}[16,17,22]$ on $\mathcal{F}(X)$ is given by

$$
d_{\infty}(f, g)=\sup _{0<\alpha \leq 1} H_{d}\left([f]^{\alpha},[g]^{\alpha}\right)=\sup _{0<\alpha \leq 1} \max \left\{\sup _{y \in[g]^{\alpha}} d\left([f]^{\alpha}, y\right), \sup _{x \in[f]^{\alpha}} d\left(x,[g]^{\alpha}\right)\right\} .
$$

Actually, $d_{\infty}$ is an extended pseudometric (for example, $d_{\infty}(f, g)=0$ where $f, g$ are the characteristic functions of $\mathbb{Q} \cap I$ and $\mathbb{I} \cap I$, respectively) although we will refer to it as a metric. Furthermore, if $d_{\infty}$ is restricted to $U S C(X)$, the family of all upper semicontinuous fuzzy sets on $X$, then we obtain an extended metric since superlevel sets are closed. 


\section{Remark 9. Notice that}

$$
\sup _{0<\alpha \leq 1} H_{d}\left([f]^{\alpha},[g]^{\alpha}\right)=\sup _{0 \leq \alpha \leq 1} H_{d}\left([f]^{\alpha},[g]^{\alpha}\right) .
$$

Indeed, it is obvious that $d_{\infty}(f, g)=\sup _{0<\alpha \leq 1} H_{d}\left([f]^{\alpha},[g]^{\alpha}\right) \leq \sup _{0 \leq \alpha \leq 1} H_{d}\left([f]^{\alpha},[g]^{\alpha}\right)$. On the other hand, suppose that $d_{\infty}(f, g)=\varepsilon$ (if $d_{\infty}(f, g)=+\infty$ the conclusion is obvious). Given $x \in[f]^{0}$ and $n \in \mathbb{N}$ we can find $\alpha_{n} \in(0,1]$ and $y \in[f]^{\alpha_{n}}$ such that $d(x, y)<1 /(2 n)$. By assumption, $H_{d}\left([f]^{\alpha_{n}},[g]^{\alpha_{n}}\right) \leq \varepsilon$ so there exists $z \in[g]^{\alpha_{n}} \subseteq[g]^{0}$ with $d(y, z) \leq \varepsilon+1 /(2 n)$. Consequently, $d(x, z)<\varepsilon+1 / n$ so $d\left(x,[g]^{0}\right)<\varepsilon+1 / n$. Hence $d\left(x,[g]^{0}\right) \leq \varepsilon$ for all $x \in[f]^{0}$.

In a similar way, it can be proved that $d\left(x,[f]^{0}\right) \leq \varepsilon$ for every $x \in[g]^{0}$. Therefore, $H_{d}\left([f]^{0},[g]^{0}\right) \leq d_{\infty}(f, g)$. by

The supremum metric can be split in two (extended) quasi-pseudometrics given

$$
\begin{aligned}
& d_{\infty}^{-}(f, g)=\sup _{0<\alpha \leq 1} e_{d}\left([f]^{\alpha},[g]^{\alpha}\right), \\
& d_{\infty}^{+}(f, g)=\sup _{0<\alpha \leq 1} e_{d}\left([g]^{\alpha},[f]^{\alpha}\right),
\end{aligned}
$$

where $e_{d}\left([f]^{\alpha},[g]^{\alpha}\right)$ is the excess of $[f]^{\alpha}$ over $[g]^{\alpha}$. We call $d_{\infty}^{-}$(resp. $\left.d_{\infty}^{+}\right)$the lower supremum quasi-pseudometric (resp. the upper supremum quasi-pseudometric). We emphasize that we use the term "quasi" because $d_{\infty}^{-}$and $d_{\infty}^{+}$are not in general symmetric. It is easy to check that $d_{\infty}(f, g)=\max \left\{d_{\infty}^{+}(f, g), d_{\infty}^{-}(f, g)\right\}$.

Moreover, it is straightforward to prove (see equation (1)) that a net $\left\{f_{\lambda}\right\}_{\lambda \in \Lambda}$ in $\mathcal{F}(X)$ is

- $d_{\infty}^{-}$-convergent to $f \in \mathcal{F}(X)$ if and only if given $\varepsilon>0$ there exists $\lambda_{\varepsilon} \in \Lambda$ such that $[f]^{\alpha} \subseteq B_{d}\left(\left[f_{\lambda}\right]^{\alpha}, \varepsilon\right)$ for all $\lambda \geq \lambda_{\varepsilon}$ and all $\alpha \in(0,1]$;

- $d_{\infty}^{+}$-convergent to $f \in \mathcal{F}(X)$ if and only if given $\varepsilon>0$ there exists $\lambda_{\varepsilon} \in \Lambda$ such that $\left[f_{\lambda}\right]^{\alpha} \subseteq B_{d}\left([f]^{\alpha}, \varepsilon\right)$ for all $\lambda \geq \lambda_{\varepsilon}$ and all $\alpha \in(0,1]$.

We will use this characterization of these two convergences in the sequel.

Remark 10. Let $(X, d)$ be a metric space and let $\left\{f_{\lambda}\right\}_{\lambda \in \Lambda}$ be a net in $\mathcal{F}(X)$. Given $f, F \in \mathcal{F}(X)$ such that $f(x) \leq \inf _{\lambda \in \Lambda} f_{\lambda}(x)$ and $F(x) \geq \sup _{\lambda \in \Lambda} f_{\lambda}(x)$ for all $x \in X$, then it is obvious that $\left\{f_{\lambda}\right\}_{\lambda \in \Lambda}$ is $d_{\infty}^{-}$-convergent (resp. $d_{\infty}^{+}$-convergent) to $f$ (resp. $F)$. In particular $\left\{f_{\lambda}\right\}_{\lambda \in \Lambda}$ is always $d_{\infty}^{-}$-convergent to the identically 0 fuzzy set and $d_{\infty}^{+}$-convergent to the constant fuzzy set 1 .

Furthermore, we know by [2, Lemma 1.5.1] that $e_{d}(A, B)=\sup _{x \in X} d(B, x)-$ $d(A, x)$ so we have that

Therefore,

$$
\begin{aligned}
& d_{\infty}^{-}(f, g)=\sup _{0<\alpha \leq 1} \sup _{x \in X} d\left([g]^{\alpha}, x\right)-d\left([f]^{\alpha}, x\right), \\
& d_{\infty}^{+}(f, g)=\sup _{0<\alpha \leq 1} \sup _{x \in X} d\left([f]^{\alpha}, x\right)-d\left([g]^{\alpha}, x\right) .
\end{aligned}
$$

$$
d_{\infty}(f, g)=\sup _{0<\alpha \leq 1} d_{u c}\left(d\left([f]^{\alpha}, \cdot\right), d\left([g]^{\alpha}, \cdot\right)\right)
$$

where $d_{u c}$ denotes the metric compatible with the topology of uniform convergence given by

$$
d_{u c}(f, g)=\sup _{x \in X}|f(x)-g(x)|
$$

The proof of the next proposition follows from the above observations. 
Proposition 2. Let $(X, d)$ be a metric space. Then a net $\left\{f_{\lambda}\right\}_{\lambda \in \Lambda}$ in $\mathcal{F}(X)$ is $d_{\infty}$-convergent to $f$ if and only if given $\varepsilon>0$ there exists $\lambda_{\varepsilon} \in \Lambda$ such that

$$
\left|d\left(\left[f_{\lambda}\right]^{\alpha}, x\right)-d\left([f]^{\alpha}, x\right)\right|<\varepsilon
$$

for all $\lambda \geq \lambda_{\varepsilon}, x \in X$ and $\alpha \in(0,1]$.

In the following, we try to give a formula which allows us to obtain, as in equations (4) and (5) for $\Gamma$-convergence, the limit of a net of functions convergent with respect to the lower or upper supremum quasi-pseudometrics. We also intend to establish a characterization of the convergence in these two quasi-pseudometrics in the spirit of Theorem 1 .

4.1. Convergence in the lower supremum quasi-pseudometric. Let $(X, d)$ be a metric space. Recall that if $f \in \mathcal{F}(X)$, then $M_{f}(A)=\sup _{a \in A} f(a)$, where $\varnothing \neq A \subseteq X$. The role of this functional in the study of the $d_{\infty}^{-}$-convergence is crucial as the next proposition shows.

Proposition 3 (cf. [14]). Let $(X, d)$ be a metric space and let $\left\{f_{\lambda}\right\}_{\lambda \in \Lambda}$ be a net in $\mathcal{F}(X)$. Consider the following statements:

(1) $\left\{f_{\lambda}\right\}_{\lambda \in \Lambda}$ is $\tau_{\leq}$-uniformly convergent to $f$;

(2) $\left\{M_{f_{\lambda}}\left(B_{d}(\cdot, \varepsilon)\right)\right\}_{\lambda \in \Lambda}$ is $\mathcal{S}_{<}$-uniformly convergent to $f$ for all $\varepsilon>0$;

(3) $\left\{f_{\lambda}\right\}_{\lambda \in \Lambda}$ is $d_{\infty}^{-}$-convergent to $f$;

(4) $\left\{M_{f_{\lambda}}\left(B_{d}(\cdot, \varepsilon)\right)\right\}_{\lambda \in \Lambda}$ is $\tau_{\leq}$-uniformly convergent to $f$ for all $\varepsilon>0$;

(5) $\left\{f_{\lambda}\right\}_{\lambda \in \Lambda}$ is $\Gamma^{-}$-convergent to $f$.

We have the following implications:

$$
\stackrel{(1)}{\searrow}(3) \Rightarrow(4) \Rightarrow(5)
$$

Proof. (1) $\Rightarrow$ (3) Suppose that $\left\{f_{\lambda}\right\}_{\lambda \in \Lambda}$ is $\tau_{\leq}$-uniformly convergent to $f$. Then there exists $\lambda_{0} \in \Lambda$ such that $f(x) \leq f_{\lambda}(x)$ for all $\lambda \geq \lambda_{0}$ and all $x \in X$. Therefore, given $\alpha \in(0,1]$ and $x \in[f]^{\alpha}$ then $\alpha \leq f(x) \leq f_{\lambda}(x)$ so $[f]^{\alpha} \subseteq B_{d}\left(\left[f_{\lambda}\right]^{\alpha}, \varepsilon\right)$ for all $\lambda \geq \lambda_{0}$. Consequently, $\left\{f_{\lambda}\right\}_{\lambda \in \Lambda}$ is $d_{\infty}^{-}$-convergent to $f$.

$(2) \Rightarrow(3)$ Let $\varepsilon>0$. By assumption, we can find $\lambda_{\varepsilon} \in \Lambda$ such that $f(x)<$ $M_{f_{\lambda}}\left(B_{d}(x, \varepsilon)\right)$ for all $\lambda \geq \lambda_{\varepsilon}$ and all $x \in X$. Consequently, given $\lambda \geq \lambda_{\varepsilon}$ and $\alpha \in(0,1]$, if $x \in[f]^{\alpha}$ we can find $x_{\lambda} \in B_{d}(x, \varepsilon)$ such that $\alpha \leq f(x) \leq f_{\lambda}\left(x_{\lambda}\right)$. Therefore, $x \in B_{d}\left(\left[f_{\lambda}\right]^{\alpha}, \varepsilon\right)$ for all $\lambda \geq \lambda_{\varepsilon}$, which concludes this implication.

$(3) \Rightarrow(4)$ Since $\left\{f_{\lambda}\right\}_{\lambda \in \Lambda}$ is $d_{\infty}^{-}$-convergent to $f$, given $\varepsilon>0$ we can find $\lambda_{\varepsilon} \in \Lambda$ such that $[f]^{f(x)} \subseteq B_{d}\left(\left[f_{\lambda}\right]^{f(x)}, \varepsilon\right)$ for all $\lambda \geq \lambda_{\varepsilon}$ and all $x \in X$ (notice that by Remark 9 there is no need to ask that $f(x)>0)$. Given $x \in X$, if $f(x)=0$ then $f(x)=0 \leq f_{\lambda}(x) \leq M_{f_{\lambda}}\left(B_{d}(x, \varepsilon)\right)$ for all $\lambda \in \Lambda$. On the other hand, if $f(x)>0$ we can find $x_{\lambda} \in B_{d}(x, \varepsilon)$ such that $f(x) \leq f_{\lambda}\left(x_{\lambda}\right)$ for all $\lambda \geq \lambda_{\varepsilon}$. Hence $f(x) \leq M_{f_{\lambda}}\left(B_{d}(x, \varepsilon)\right)$ for all $\lambda \geq \lambda_{\varepsilon}$ and all $x \in X$.

$(4) \Rightarrow(5)$ Given $\varepsilon>0$ then $f(x) \leq M_{f_{\lambda}}\left(B_{d}(x, \varepsilon)\right)$ for all $\lambda \geq \lambda_{\varepsilon}$ and all $x \in X$. Hence we deduce that $f(x) \leq \inf _{\lambda \geq \lambda_{\varepsilon}} M_{f_{\lambda}}\left(B_{d}(x, \varepsilon)\right)$ for all $x \in X$. Consequently, $f(x) \leq \inf _{\varepsilon>0} \liminf \operatorname{in}_{\lambda \in \Lambda} M_{f_{\lambda}}\left(B_{d}(x, \varepsilon)\right)$ so $\left\{f_{\lambda}\right\}_{\lambda \in \Lambda}$ is $\Gamma^{-}$-convergent to $f$. 
Remark 11. One of the main uses of the $\Gamma$-convergence appears in the context of variational analysis where questions about the maximum or minimum of a function relative to a certain set are fundamental. In this way, a number of basic questions with respect to the stability of the set of minimizers or maximizers appear in a natural way. For example, if a net $\left\{f_{\lambda}\right\}_{\lambda \in \Lambda}$ converges to $f$ in some sense does the net $\left\{M_{f_{\lambda}}(X)\right\}_{\lambda \in \Lambda}$ converge to $M_{f}(X)$ ? If $x_{\lambda} \in \operatorname{Argmax}_{X} f_{\lambda}=\{x \in X: f(x)=$ $\left.M_{f_{\lambda}}(X)\right\}$ for all $\lambda \in \Lambda$ and $\left\{x_{\lambda}\right\}_{\lambda \in \Lambda}$ converges to $x$, is $x$ in $\operatorname{Argmax}_{X} f$ ? The dual version of these questions for minimizers and others are developed deeply in [24]. Although these results deal with lower semicontinuous functions, it is not difficult to translate them to the context of fuzzy sets.

For example, it is not hard to prove that (cf. [24, Proposition 7.29 (b)]) a net of fuzzy sets $\left\{f_{\lambda}\right\}_{\lambda \in \Lambda}$ is $\Gamma^{-}$-convergent to $f$ if and only if $M_{f}\left(B_{d}(x, \varepsilon)\right) \leq$ $\liminf _{\lambda \in \Lambda} M_{f_{\lambda}}\left(B_{d}(x, \varepsilon)\right)$ for every $x \in X$ and $\varepsilon>0$. Hence $M_{f}(X) \leq \liminf \inf _{\lambda \in \Lambda} M_{f_{\lambda}}(X)$ (cf. [2, Proposition 1.3.5]).

The previous proposition gives a result in this direction when considering the $d_{\infty}^{-}$convergence. In this way, from (4) we deduce that $f(x) \leq \liminf _{\lambda \in \Lambda} M_{f_{\lambda}}\left(B_{d}(x, \varepsilon)\right)$ for all $x \in X$ and all $\varepsilon>0$ and $M_{f}(X) \leq M_{f_{\lambda}}(X)$ residually. Taking into account this and the above comments, we can easily deduce that $d_{\infty}^{-}$convergence implies $\Gamma^{-}$-convergence.

At this point, it is natural to wonder whether it is possible to obtain a characterization of the $d_{\infty}^{-}$-convergence similar to the characterization of the $\Gamma^{-}$-convergence that we have just mentioned. We prove it in Corollary 4.

From this, it is also easy to obtain $(4) \Rightarrow(5)$ of the above proposition.

Now we provide several examples showing that the implications in the above proposition cannot be reversed in general. In all of them, we will consider that the real line and its subsets are endowed with the Euclidean metric $d_{e}$.

Example $2((3) \nRightarrow(1))$. Consider the sequence of fuzzy sets on I given by

$$
f_{n}(x)=\left\{\begin{array}{ll}
x-\frac{1}{3 n} & \text { if } \frac{1}{3 n}<x<1-\frac{1}{3 n} \\
1 & \text { otherwise }
\end{array},\right.
$$

for all $n \in \mathbb{N}$. Let $f$ be the identity function on $I$. Given $\varepsilon>0$, take $n_{\varepsilon} \in \mathbb{N}$ with $1 / n_{\varepsilon}<\varepsilon$. Let $\alpha \in(0,1]$ and $x \in[f]^{\alpha}$, i. e. $\alpha \leq f(x)=x$. Pick $n \geq n_{\varepsilon}$. If $x+1 /(3 n) \in[0,1]$ then $\alpha \leq x \leq f_{n}(x+1 /(3 n))$. Since $x+1 /(3 n) \in B_{d_{e}}(x, \varepsilon)$ then $x \in B_{d_{e}}\left(\left[f_{n}\right]^{\alpha}, \varepsilon\right)$. If $x+1 /(3 n) \notin I$ then $f_{n}(x)=1 \geq \alpha$ so $x \in B_{d_{e}}\left(\left[f_{n}\right]^{\alpha}, \varepsilon\right)$. Consequently, $\left\{f_{n}\right\}_{n \in \mathbb{N}}$ is $d_{\infty}^{-}$-convergent to $f$.

Nevertheless, the sequence $\left\{f_{n}\right\}_{n \in \mathbb{N}}$ is obviously not $\tau_{\leq}$-uniformly convergent to $f$.

Example $3((3) \nRightarrow(2))$. Just consider the constant sequence $\left\{f_{n}\right\}_{n \in \mathbb{N}}$ of fuzzy sets on $\mathbb{R}$ such that $f_{n}$ is the function identically 1 . Then $f_{n}(x)=M_{f_{n}}\left(B_{d}(x, \varepsilon)\right)=1$ for all $x \in \mathbb{R}$ and all $\varepsilon>0$. It is obvious that $\left\{f_{n}\right\}_{n \in \mathbb{N}}$ is $d_{\infty}^{-}$-convergent to the constant fuzzy set 1 but $\left\{M_{f_{n}}\left(B_{d_{e}}(\cdot, \varepsilon)\right)\right\}_{n \in \mathbb{N}}$ is not $\mathcal{S}_{<- \text {-pointwise convergent to that }}$ fuzzy set for any $\varepsilon>0$.

Example $4((4) \nRightarrow(3)$ and $(5) \nRightarrow(3))$. Let us consider the space $\ell_{1}$ of all real sequences such that $\|x\|_{1}=\sum_{n=1}^{\infty}|x(n)|<\infty$. Let $\left\{f_{n}\right\}_{n \in \mathbb{N}}$ be the sequence of fuzzy sets on $\ell_{1}$ given by

$$
f_{n}(x)=\left\{\begin{array}{ll}
1-\frac{1}{k} & \text { if } x=\frac{1}{n} e_{k} \\
0 & \text { otherwise }
\end{array},\right.
$$


where $e_{k}$ is the sequence whose terms are always 0 except the $k^{\text {th }}$ term which is 1 , for all $k \in \mathbb{N}$. Let $f$ be the characteristic function of the constant zero sequence $\mathbf{0}$. It is easy to see that $f_{n}$ is upper semicontinuous for all $n \in \mathbb{N}$ and $f$ so is. It is clear that $\left\{M_{f_{n}}\left(B_{\|\cdot\|_{1}}(\cdot, \varepsilon)\right)\right\}_{n \in \mathbb{N}}$ is $\tau_{\leq}$-uniformly convergent to $f$ for all $\varepsilon>0$. In fact, given $\varepsilon>0$, let $n_{0} \in \mathbb{N}$ such that $1 / n_{0}<\varepsilon$. Then, given $x \in \ell_{1}$ we have that

$$
\begin{cases}f(x)=0 \leq f_{n}(x) \leq M_{f_{n}}\left(B_{\|\cdot\|_{1}}(x, \varepsilon)\right) & \text { if } x \neq \mathbf{0} \\ f(x) \leq M_{f_{n}}\left(B_{\|\cdot\|_{1}}(x, \varepsilon)\right)=M_{f_{n}}\left(\left\{\frac{1}{n} e_{k}: k \in \mathbb{N}\right\}\right)=1 & \text { if } x=\mathbf{0}\end{cases}
$$

for all $n \geq n_{0}$. Nevertheless, $\left\{f_{n}\right\}_{n \in \mathbb{N}}$ is not $d_{\infty}^{-}$-convergent to $f$ since $[f]^{1}=\{\mathbf{0}\}$ but $\left[f_{n}\right]^{1}=\varnothing$ for all $n \in \mathbb{N}$.

Example $5((5) \nRightarrow(4))$. Let us define on $\mathbb{R}$ the sequence of fuzzy sets $\left\{f_{n}\right\}_{n \in \mathbb{N}}$ where

$$
f_{n}(x)=\left\{\begin{array}{ll}
\frac{n-1}{n^{3}}\left(n^{2}-x^{2}\right) & \text { if }-n \leq x \leq n \\
0 & \text { otherwise }
\end{array} .\right.
$$

It is not difficult to see that

$$
\inf _{\varepsilon>0} \liminf _{n \rightarrow+\infty} M_{f_{n}}\left(B_{d_{e}}(a, \varepsilon)\right)=\liminf _{n \rightarrow+\infty} M_{f_{n}}\left(B_{d_{e}}(a, \delta)\right)=1
$$

for all $a \in \mathbb{R}$ and all $\delta>0$. Therefore, $\left\{f_{n}\right\}_{n \in \mathbb{N}}$ is $\Gamma^{-}$-convergent to the constant function $f(x)=1$ for all $x \in \mathbb{R}$. Nevertheless, given $\varepsilon>0$ the sequence

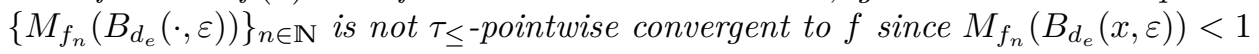
for all $n \in \mathbb{N}$ and all $x \in \mathbb{R}$.

The next two examples show that, in general, there is no relationship between the lower uniform convergence and the $d_{\infty}^{-}$-convergence.

Example 6. Let us consider the sequence $\left\{f_{n}\right\}_{n \in \mathbb{N}}$ of fuzzy sets on $\mathbb{R}$ given by $f_{n}(x)=1-1 / n$ for all $n \in \mathbb{N}$ and all $x \in \mathbb{R}$. Then it is obvious that this sequence is (lower-) uniformly convergent to the constant fuzzy set $f(x)=1$ for all $x \in \mathbb{R}$. However, $\left\{f_{n}\right\}_{n \in \mathbb{N}}$ is not $d_{\infty}^{-}$-convergent to $f$ since $[f]^{1}=\mathbb{R} \nsubseteq B_{d}\left(\left[f_{n}\right]^{1}, \varepsilon\right)=\varnothing$ for all $\varepsilon>0$ and all $n \in \mathbb{N}$.

We now exhibit that $d_{\infty}^{-}$-convergence does not imply lower uniform convergence (compare with Proposition 5).

Example 7. In the real line, consider, for all $n \in \mathbb{N}$, the following fuzzy sets:

$$
f_{n}(x)= \begin{cases}-n \frac{x}{2}+\frac{1}{2} & \text { if }-\frac{1}{n} \leq x<0 \\ n \frac{x}{2}+\frac{1}{2} & \text { if } 0 \leq x \leq \frac{1}{n} \\ 1 & \text { otherwise }\end{cases}
$$

Let $f(x)=1$ for all $x \in \mathbb{R}$. Then, given $\varepsilon>0$ choose $n_{\varepsilon} \in \mathbb{N}$ such that $1 / n_{\varepsilon}<\varepsilon / 2$. Given $x \in \mathbb{R}$, if $x \notin\left[-\frac{1}{n_{\varepsilon}}, \frac{1}{n_{\varepsilon}}\right]$ then $f_{n}(x)=1$ for all $n \geq n_{\varepsilon}$. If $x \in\left[-\frac{1}{n}, \frac{1}{n}\right]$ for some $n \geq n_{\varepsilon}$ then $x-\varepsilon / 2 \notin\left[-\frac{1}{n}, \frac{1}{n}\right]$ or $x+\varepsilon / 2 \notin\left[-\frac{1}{n}, \frac{1}{n}\right]$. In any case, given $n \geq n_{\varepsilon}$, $f_{n}\left(x^{\prime}\right)=1$ for some $x^{\prime} \in B_{d_{e}}(x, \varepsilon)$. This proves that $\left\{f_{n}\right\}_{n \in \mathbb{N}}$ is $d_{\infty}^{-}$-convergent to $f$.

However, this sequence is not lower uniformly convergent to $f$, since $f_{n}(0)=1 / 2$ for all $n \in \mathbb{N}$ but $f(0)=1$. 
Corollary 1. Let $(X, d)$ be a metric space and let $\left\{f_{\lambda}\right\}_{\lambda \in \Lambda}$ be a net in $\mathcal{F}(X)$. If $\left\{f_{\lambda}\right\}_{\lambda \in \Lambda}$ is $d_{\infty}^{-}$-convergent to $f$ then, given $x \in X$,

$$
f(x) \leq \inf _{\varepsilon>0} \liminf _{\lambda \in \Lambda} M_{f_{\lambda}}\left(B_{d}(x, \varepsilon)\right) .
$$

Proof. This is a direct consequence of Proposition 3 and equation 4.

In [14, Lemma 3.3] it is proved a nice characterization for the convergence in the supremum metric of sequences of upper semicontinuous fuzzy sets with compact support, which can be considered as a bilateral converse of implication $(3) \Rightarrow(4)$ in Proposition 3. We can reformulate that result for the lower supremum quasipseudometric in our terms in the following way. Given a metric space $(X, d)$, let us denote by $S U S C(X)$ the family of all upper semicontinuous fuzzy sets on $X$ with compact support. Then the result asserts that a sequence $\left\{f_{n}\right\}_{n \in \mathbb{N}}$ in $S U S C(X)$ is $d_{\infty}^{-}$-convergent to $f \in S U S C(X)$ if and only if $\left\{M_{f_{n}}\left(\overline{B_{d}}(x, \varepsilon)\right)\right\}_{n \in \mathbb{N}}$ is $\tau_{\leq}$-uniformly convergent to $f$ for all $\varepsilon>0$. Therefore, this lemma shows that the converse of implication $(3) \Rightarrow(4)$ of Proposition 3 is true under these additional hypotheses. Nevertheless, this result is not valid in general as Example 4 exhibits. Notice that in this example, the sequence $\left\{f_{n}\right\}_{n \in \mathbb{N}}$ is $d_{\infty}^{-}$-convergent to every fuzzy set $f$ verifying that end $f \subseteq\left[\left(\ell_{1}-\{\mathbf{0}\}\right) \times\{0\}\right] \cup[\{\mathbf{0}\} \times[0,1[)]=A$. However, the set $A$ does not correspond to the endograph of a fuzzy set. This shows that there is no hope to obtain a characterization of the $d_{\infty}^{-}$-convergence in the spirit of the characterization of the $\Gamma^{-}$-convergence given by equation (4) in Section 3.2.

However, we can provide a purely set-theoretic characterization of the $d_{\infty}^{-}$convergence. To achieve this, we need to introduce an apropriate concept of uniform convergence for sets.

Let $X$ be a nonempty set and let $\mathcal{P}(X)$ be the family of all subsets of $X$. Recall that a net $\left\{A_{\lambda}\right\}_{\lambda \in \Lambda}$ in $\mathcal{P}(X)$ is lower convergent to a set $A \in \mathcal{P}(X)$ if

$$
A \subseteq \liminf _{\lambda \in \Lambda} A_{\lambda}:=\bigcup_{\lambda_{0} \in \Lambda}\left(\bigcap_{\lambda \geq \lambda_{0}} A_{\lambda}\right),
$$

that is, we can find $\lambda_{0} \in \Lambda$ such that

$$
A \subseteq \cap_{\lambda \geq \lambda_{0}} A_{\lambda} .
$$

Notice that if $(X, \tau)$ is a topological space then $\liminf \operatorname{in}_{\lambda \in \Lambda} A_{\lambda} \subseteq \operatorname{Li} A_{\lambda}$ and the two limits coincide when $\tau$ is the discrete topology.

The previous set-convergence allows us to define a convergence for a net of multifunctions. The following definition seems to be a natural uniform version for this convergence of multifunctions.

Definition 10. Let $X$ be a nonempty set and let $\left\{f_{\lambda}\right\}_{\lambda \in \Lambda}$ be a net of multifunctions $f_{\lambda}: X \rightrightarrows I$. We say that the net $\left\{f_{\lambda}\right\}_{\lambda \in \Lambda}$ is lower uniformly convergent to the multifunction $f: X \rightrightarrows I$ if we can find $\lambda_{0} \in \Lambda$ such that

$$
f(x) \subseteq \bigcap_{\lambda \geq \lambda_{0}} f_{\lambda}(x) \text { for all } x \in X .
$$

The following result characterizes $d_{\infty}^{-}$-convergence in the most general context.

Theorem 2. Let $(X, d)$ be a metric space and let us consider that $I$ is endowed with the Alexandroff topology $\tau_{\leq}$. Then a net $\left\{f_{\lambda}\right\}_{\lambda \in \Lambda}$ in $\mathcal{F}(X)$ is $d_{\infty}^{-}$-convergent 
to a fuzzy set $f$ if and only if the net of multifunctions $\left\{f_{\lambda}^{\varepsilon}\right\}_{\lambda \in \Lambda}$ is lower uniformly convergent to $f$ for all $\varepsilon>0$, where $f_{\lambda}^{\varepsilon}: X \rightrightarrows I$ is given by $f_{\lambda}^{\varepsilon}(x)={\overline{f_{\lambda}\left(B_{d}(x, \varepsilon)\right)}}^{\tau} \leq$.

Proof. Suppose that $\left\{f_{\lambda}\right\}_{\lambda \in \Lambda}$ is $d_{\infty}^{-}$-convergent to $f$. Let $\varepsilon>0$. Given $x \in X$, if $f(x)=0$ it is obvious that $f(x) \in{\overline{f_{\lambda}\left(B_{d}(x, \varepsilon)\right)}}^{\tau}=\downarrow f_{\lambda}\left(B_{d}(x, \varepsilon)\right)$ for all $\lambda \in \Lambda$ (see Remark 3). Otherwise, by assumption, there exists $\lambda_{\varepsilon} \in \Lambda$ such that $[f]^{\alpha} \subseteq$ $B_{d}\left(\left[f_{\lambda}\right]^{\alpha}, \varepsilon\right)$ for all $\lambda \geq \lambda_{\varepsilon}$ and all $\alpha \in(0,1]$. In particular, taking $\alpha=f(x)>0$, there exists $x_{\lambda} \in B_{d}(x, \varepsilon)$ such that $f(x) \leq f_{\lambda}\left(x_{\lambda}\right)$ for all $\lambda \geq \lambda_{\varepsilon}$. Hence $f(x) \in$ $\cap_{\lambda \geq \lambda_{\varepsilon}}{\overline{f_{\lambda}\left(B_{d}(x, \varepsilon)\right)}}^{\tau} \leq$. Since $\lambda_{\varepsilon}$ does not depend on $x$, we have proved the necessity.

Conversely, let $\varepsilon>0$ and $\alpha \in(0,1]$. Suppose that $x_{0} \in[f]^{\alpha}$. By hypothesis, there exists $\lambda_{\varepsilon} \in \Lambda$ such that $f(x) \subseteq \cap_{\lambda \geq \lambda_{\varepsilon}}{\overline{f_{\lambda}\left(B_{d}(x, \varepsilon)\right)}}^{\tau} \leq$ for all $x \in X$. In particular, $f\left(x_{0}\right) \in{\overline{f_{\lambda}\left(B_{d}\left(x_{0}, \varepsilon\right)\right)}}^{\tau} \leq$ for all $\lambda \geq \lambda_{\varepsilon}$ so $x_{0} \in B_{d}\left(\left[f_{\lambda}\right]^{\alpha}, \varepsilon\right)$ for all $\lambda \geq \lambda_{\varepsilon}$ which finishes the proof.

Corollary 2 (cf. Equation 4). Let $(X, d)$ be a metric space. If $\left\{f_{\lambda}\right\}_{\lambda \in \Lambda}$ is a net in $\mathcal{F}(X) d_{\infty}^{-}$-convergent to $f$ then

$$
f(x) \in \bigcap_{\varepsilon>0} \liminf _{\lambda \in \lambda}{\overline{f_{\lambda}\left(B_{d}(x, \varepsilon)\right.}}^{\tau} \leq \text { for all } x \in X .
$$

Remark 12. Given a net $\left\{f_{\lambda}\right\}_{\lambda \in \Lambda}$ in $\mathcal{F}(X)$, it is easy to check that

$$
\sup \bigcap_{\varepsilon>0} \liminf _{\lambda \in \lambda}{\overline{f_{\lambda}\left(B_{d}(x, \varepsilon)\right)^{\tau}}}^{\tau}=\inf _{\varepsilon>0} \liminf _{\lambda \in \Lambda} M_{f_{\lambda}}\left(B_{d}(x, \varepsilon)\right)
$$

from which we again deduce immediately that $d_{\infty}^{-}$-convergence implies $\Gamma^{-}$-convergence.

Corollary 3 (cf. [14, Lemma 3.3]). Let $(X, d)$ be a metric space. Then a net $\left\{f_{\lambda}\right\}_{\lambda \in \Lambda}$ in $S U S C(X)$ is $d_{\infty}^{-}$-convergent to $f \in S U S C(X)$ if and only if $\left\{M_{f_{\lambda}}\left(B_{d}(\cdot, \varepsilon)\right)\right\}_{\lambda \in \Lambda}$ is $\tau_{\leq}$-uniformly convergent to $f$ for all $\varepsilon>0$.

Proof. By Proposition 3, we only have to prove the sufficiency.

Let $\varepsilon>0$. By assumption, we can find $\lambda_{\varepsilon}$ such that $f(x) \leq M_{f_{\lambda}}\left(B_{d}(x, \varepsilon / 2)\right)$ for all $\lambda \geq \lambda_{\varepsilon}$ and all $x \in X$. We show that $[f]^{\alpha} \subseteq B_{d}\left(\left[f_{\lambda}\right]^{\alpha}, \varepsilon\right)$ for all $\lambda \geq \lambda_{\varepsilon}$ and all $\alpha \in(0,1]$. Let $x \in[f]^{\alpha}$. Given $\lambda \geq \lambda_{\varepsilon}$, if $\alpha \leq f(x)<M_{f_{\lambda}}\left(B_{d}(x, \varepsilon / 2)\right)$ then it is obvious that $x \in B_{d}\left(\left[f_{\lambda}\right]^{\alpha}, \varepsilon\right)$. Otherwise, suppose that $f(x)=M_{f_{\lambda}}\left(B_{d}(x, \varepsilon / 2)\right)>0$ (if $f(x)=0$ the conclusion is obvious). Then we can find a sequence $\left\{a_{n}\right\}_{n \in \mathbb{N}}$ in $B_{d}(x, \varepsilon / 2)$ such that $f_{\lambda}\left(a_{n}\right)>0$ for all $n \in \mathbb{N}$ and $\lim _{n \rightarrow+\infty} f_{\lambda}\left(a_{n}\right)=f(x)$. Since $\left[f_{\lambda}\right]^{0}$ is compact, we can suppose without loss of generality that the sequence $\left\{a_{n}\right\}_{n \in \mathbb{N}}$ is convergent to $a \in \overline{B_{d}}(x, \varepsilon / 2)$. Since $f_{\lambda}$ is upper semicontinuous we have that $\lim \sup _{n \rightarrow+\infty} f_{\lambda}\left(a_{n}\right)=f(x) \leq f_{\lambda}(a)$. We conclude again that $x \in B_{d}\left(\left[f_{\lambda}\right]^{\alpha}, \varepsilon\right)$. Consequently, $\left\{f_{\lambda}\right\}_{\lambda \in \Lambda}$ is $d_{\infty}^{-}$-convergent to $f$.

Corollary 4. Let $(X, d)$ be a metric space. Then a net $\left\{f_{\lambda}\right\}_{\lambda \in \Lambda}$ in $\mathcal{F}(X)$ is $d_{\infty}^{-}$convergent to $f$ if and only if for each $\varepsilon>0$ there exists $\lambda_{\varepsilon} \in \Lambda$ such that

$$
f(x) \leq M_{f_{\lambda}}\left(B_{d}(x, \varepsilon)\right) \text { for all } \lambda \geq \lambda_{\varepsilon} \text { and all } x \in X
$$

and

$$
\text { if given } \lambda \geq \lambda_{\varepsilon}, f(x)=M_{f_{\lambda}}\left(B_{d}(x, \varepsilon)\right) \text { then } \operatorname{Argmax}_{B_{d}(x, \varepsilon)} f_{\lambda} \neq \varnothing \text {. }
$$

Proof. It easily follows from Theorem 2.

For convergence of sequences, we can give the following characterization of the $d_{\infty}^{-}$-convergence (cf. Theorem 1). 
Theorem 3. Let $(X, d)$ be a metric space and let $\left\{f_{n}\right\}_{n \in \mathbb{N}}$ be a sequence in $\mathcal{F}(X)$. Then $\left\{f_{n}\right\}_{n \in \mathbb{N}}$ is $d_{\infty}^{-}$-convergent to a fuzzy set $f$ if and only if there exists a sequence $\left\{g_{n}\right\}_{n \in \mathbb{N}}$ of self-maps in $X$ uniformly convergent to $i d_{X}$ such that $\left\{f_{n} \circ g_{n}\right\}_{n \in \mathbb{N}}$ is $\tau_{\leq}$-uniformly convergent to $f$.

Proof. Suppose first that $\left\{f_{n}\right\}_{n \in \mathbb{N}}$ is $d_{\infty}^{-}$-convergent to $f$. Therefore, given $k \in \mathbb{N}$ we can find $n_{k} \in \mathbb{N}$ such that $[f]^{f(x)} \subseteq B_{d}\left(\left[f_{n}\right]^{f(x)}, 1 / k\right)$ for all $n \geq n_{k}$ and all $x \in X$. We can assume without loss of generality that the sequence $\left\{n_{k}\right\}_{k \in \mathbb{N}}$ is strictly increasing. Given $x \in[f]^{0}$, since $x \in[f]^{f(x)}$ (see Remark 9), for each $n \geq n_{k}$ pick $x_{n k} \in B_{d}(x, 1 / k)$ such that $f(x) \leq f_{n}\left(x_{n k}\right)$. For each $n \in \mathbb{N}$, define $g_{n}(x)=x_{n k}$ when $n_{k} \leq n<n_{k+1}$ and $g_{n}(x)=x_{0}$ when $n<n_{1}$ for some fixed point $x_{0} \in X$. On the other hand, if $x \notin[f]^{0}$ define $g_{n}(x)=x$ for all $n \in \mathbb{N}$. Then $\left\{g_{n}\right\}_{n \in \mathbb{N}}$ is the desired sequence. In fact, given $k \in \mathbb{N}$ then $d\left(x, g_{n}(x)\right) \leq d\left(x, x_{n k}\right)<1 / k$ for all $n \geq n_{k}$ and all $x \in X$ so $\left\{g_{n}\right\}_{n \in \mathbb{N}}$ is uniformly convergent to $i d_{X}$. Furthermore, $f(x) \leq f_{n}\left(g_{n}(x)\right)$ for all $n \geq n_{k}$ and all $x \in X$.

Conversely, let $\varepsilon>0, \alpha \in(0,1]$ and $x_{0} \in X$ such that $\alpha \leq f\left(x_{0}\right)$. By assumption, there exists $n_{\varepsilon} \in \mathbb{N}$ such that $d\left(x, g_{n}(x)\right)<\varepsilon$ and $f(x) \leq f_{n}\left(g_{n}(x)\right)$ for all $n \geq n_{\varepsilon}$ and all $x \in X$. This means that $x_{0} \in B_{d}\left(\left[f_{n}\right]^{\alpha}, \varepsilon\right)$ for all $n \geq n_{\varepsilon}$. Since $n_{\varepsilon}$ does not depend on $\alpha$ the proof is finished.

Remark 13. By Theorem 1 we deduce that a sequence $\left\{f_{n}\right\}_{n \in \mathbb{N}}$ of fuzzy sets in a first countable topological space is $\Gamma^{-}$-convergent to a fuzzy set $f$ if and only if there exists a sequence $\left\{g_{n}\right\}_{n \in \mathbb{N}}$ of self-maps on $X$ pointwise convergent to $i d_{X}$ such that $\left\{f_{n} \circ g_{n}\right\}_{n \in \mathbb{N}}$ is lower pointwise convergent to $f$. From this and the above result, we obtain again that $d_{\infty}^{-}$-convergence of sequences implies $\Gamma^{-}$-convergence.

4.2. Convergence in the upper supremum quasi-pseudometric. Now, we center our attention into the upper part.

Proposition 4. Let $(X, d)$ be a metric space and let $\left\{f_{\lambda}\right\}_{\lambda \in \Lambda}$ be a net in $\mathcal{F}(X)$. Consider the following statements:

(1) $\left\{f_{\lambda}\right\}_{\lambda \in \Lambda}$ is $\mathcal{S}_{>}$-uniformly convergent to $M_{f}\left(B_{d}(\cdot, \varepsilon)\right)$ for all $\varepsilon>0$;

(2) $\left\{f_{\lambda}\right\}_{\lambda \in \Lambda}$ is $d_{\infty}^{+}$-convergent to $f$;

(3) $\left\{f_{\lambda}\right\}_{\lambda \in \Lambda}$ is $\tau_{\geq}$-uniformly convergent to $M_{f}\left(B_{d}(\cdot, \varepsilon)\right)$ for all $\varepsilon>0$.

Then the following implications hold:

$$
(1) \Rightarrow(2) \Rightarrow(3) \text {. }
$$

Proof. (1) $\Rightarrow(2)$ Given $\varepsilon>0$ there exists $\lambda_{\varepsilon} \in \Lambda$ such that $f_{\lambda}(x)<M_{f}\left(B_{d}(x, \varepsilon)\right)$ for all $\lambda \geq \lambda_{\varepsilon}$ and all $x \in X$. Let $\alpha \in(0,1]$. Given $\lambda \geq \lambda_{\varepsilon}$, if $x \in\left[f_{\lambda}\right]^{\alpha}$ then $\alpha \leq f_{\lambda}(x)<M_{f}\left(B_{d}(x, \varepsilon)\right)$ so we can find $x_{\lambda} \in B_{d}(x, \varepsilon)$ such that $f_{\lambda}(x)<f\left(x_{\lambda}\right)$. Consequently, $x \in B_{d}\left([f]^{\alpha}, \varepsilon\right)$. Therefore, $\left\{f_{\lambda}\right\}_{\lambda \in \Lambda}$ is $d_{\infty}^{+}$-convergent to $f$.

(2) $\Rightarrow$ (3) If $\left\{f_{\lambda}\right\}_{\lambda \in \Lambda}$ is $d_{\infty}^{+}$-convergent to $f$ then, given $\varepsilon>0$ we can find $\lambda_{\varepsilon} \in \Lambda$ such that $\left[f_{\lambda}\right]^{\alpha} \subseteq B_{d}\left([f]^{\alpha}, \varepsilon\right)$ for all $\lambda \geq \lambda_{\varepsilon}$ and all $\alpha \in(0,1]$. Fix $x \in X$ and $\lambda \geq \lambda_{\varepsilon}$. If $f_{\lambda}(x)=0$ it is clear that $f_{\lambda}(x) \leq M_{f}\left(B_{d}(x, \varepsilon)\right)$. If $f_{\lambda}(x)>0$, then $\left[f_{\lambda}\right]^{f_{\lambda}(x)} \subseteq B_{d}\left([f]^{f_{\lambda}(x)}, \varepsilon\right)$ so $f_{\lambda}(x) \leq M_{f}\left(B_{d}(x, \varepsilon)\right)$, which concludes the proof.

Remark 14. Observe that if $\left\{f_{\lambda}\right\}_{\lambda \in \Lambda}$ is $d_{\infty}^{+}$-convergent to $f$ and $x$ is a local maximum of $f$ then $\left\{f_{\lambda}(x)\right\}_{\lambda \in \Lambda}$ is $\tau_{\geq}$-convergent to $f(x)$. In fact, we can find $\varepsilon>0$ such that $M_{f}\left(B_{d}(x, \varepsilon)\right)=f(x)$ and, by (3) of the above result, $\lambda_{0} \in \Lambda$ such that

$$
f_{\lambda}(x) \leq M_{f}\left(B_{d}(x, \varepsilon)\right)=f(x),
$$


for all $\lambda \geq \lambda_{0}$ so the conclusion follows.

In particular, if $f$ is a constant fuzzy set then $\left\{f_{\lambda}\right\}_{\lambda \in \Lambda}$ is $d_{\infty}^{+}$-convergent to $f$ if

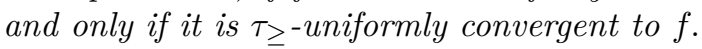

It is clear that the converse of implication $(1) \Rightarrow(2)$ of the above proposition is not true in general since $\mathcal{S}_{>}$-uniform convergence is not topological but $d_{\infty}^{+}$convergence so is (just consider the constant sequence whose elements are the zero function). Furthermore, $(2) \Rightarrow(3)$ cannot be reversed either as the next example shows.

Example 8. For each $n \in \mathbb{N}$ define the fuzzy sets $f_{n}(x)=1-x$ for all $x \in I$ and

$$
f(x)=\left\{\begin{array}{ll}
1-x & \text { if } x \neq 0 \\
0 & \text { if } x=0
\end{array} .\right.
$$

It is easy to see that $\left\{f_{n}\right\}_{n \in \mathbb{N}}$ is $\tau_{\geq}$-uniformly convergent to $M_{f}\left(B_{d}(\cdot, \varepsilon)\right)$ for all $\varepsilon>0$ (however, observe that $\left\{f_{n}(0)\right\}_{n \in \mathbb{N}}$ is not convergent to $f(0)$ ). Nevertheless, $\left[f_{n}\right]^{1}=\{0\}$ for all $n \in \mathbb{N}$ and $[f]^{1}=\varnothing$. Consequently, $\left\{f_{n}\right\}_{n \in \mathbb{N}}$ is not $d_{\infty}^{+}$-convergent to $f$.

Remark 15. Observe that by statement (3) of Proposition 4 , if $\left\{f_{\lambda}\right\}_{\lambda \in \Lambda}$ is $d_{\infty}^{+}$convergent to $f$ then

$$
\limsup _{\lambda \in \Lambda} f_{\lambda}(x) \leq \bar{f}(x)=\limsup _{x^{\prime} \rightarrow x} f\left(x^{\prime}\right),
$$

for all $x \in X$. Nevertheless, we cannot obtain the converse (see Example 10).

Furthermore, we can also deduce that $M_{f_{\lambda}}(X) \leq M_{f}(X)$ residually.

Remark 16. Notice that to obtain the equivalence between (2) and (3) of the above proposition we need to consider at least those fuzzy sets $f$ which verify that given $x \in X$ if the net $\left\{M_{f}\left(B_{d}(x, \delta)\right)\right\}_{\delta>0}$ is residually constant then the net $\left\{\operatorname{Argmax}_{B_{d}(x, \delta)} f\right\}_{\delta>0}$ is residually nonempty (see Example 8). Otherwise, we can find $x_{0} \in X$ and $\varepsilon_{0}>0$ such that $\operatorname{Argmax}_{B_{d}\left(x_{0}, \varepsilon_{0}\right)} f=\varnothing$ but for every $\varepsilon>0$ there exists $\varepsilon>\delta_{\varepsilon}>0$ such that $M_{f}\left(B_{d}\left(x_{0}, \delta_{\varepsilon}\right)\right)=M_{f}\left(B_{d}\left(x_{0}, \varepsilon_{0}\right)\right)$. For each $\delta>0$ define $f_{\delta}$ by $f_{\delta}(x)=M_{f}\left(B_{d}(x, \delta)\right)$ for all $x \in X$. It is clear that $\left\{f_{\delta}\right\}_{\delta>0}$ is $\tau_{\geq}$-uniformly convergent to $M_{f}\left(B_{d}(\cdot, \varepsilon)\right)$ for all $\varepsilon>0$. Nevertheless, if $\left\{f_{\delta}\right\}_{\delta>0}$ is $d_{\infty}^{+-}$-convergent to $f$ then we can find $\gamma_{\varepsilon_{0}}>0$ such that $\left[f_{\delta}\right]^{\alpha} \subseteq B_{d}\left([f]^{\alpha}, \varepsilon_{0}\right)$ for all $\delta \leq \gamma_{\varepsilon_{0}}$ and for all $\alpha \in(0,1]$. However, $x_{0} \in\left[f_{\delta_{\gamma_{0}}}\right]^{M_{f}\left(B_{d}\left(x_{0}, \delta_{\gamma_{0}}\right)\right)}$ but since $\operatorname{Argmax}_{B_{d}\left(x_{0}, \varepsilon_{0}\right)} f=\varnothing$ and $M_{f}\left(B_{d}\left(x_{0}, \varepsilon_{0}\right)\right)=M_{f}\left(B_{d}\left(x_{0}, \delta_{\gamma_{\varepsilon_{0}}}\right)\right)$ then $x_{0} \notin B_{d}\left([f]^{M_{f}\left(B_{d}\left(x_{0}, \delta_{\gamma_{0}}\right)\right.}, \varepsilon_{0}\right)$.

Proposition 5. Let $(X, d)$ be a metric space, let $\left\{f_{\lambda}\right\}_{\lambda \in \Lambda}$ be a net in $\mathcal{F}(X)$ and let $f \in U U S C(X)$. Consider the following statements:

(1) $\left\{f_{\lambda}\right\}_{\lambda \in \Lambda}$ is $\tau_{\geq}$-uniformly convergent to $f$;

(2) $\left\{f_{\lambda}\right\}_{\lambda \in \Lambda}$ is $d_{\infty}^{+}$-convergent to $f$;

(3) $\left\{f_{\lambda}\right\}_{\lambda \in \Lambda}$ is upper uniformly convergent to $f$;

(4) $\left\{f_{\lambda}\right\}_{\lambda \in \Lambda}$ is $\Gamma^{+}$-convergent to $f$.

Then the following implications hold:

$$
(1) \Rightarrow(2) \Rightarrow(3) \Rightarrow(4) \text {. }
$$

Proof. (1) $\Rightarrow$ (2) If $\left\{f_{\lambda}\right\}_{\lambda \in \Lambda}$ is $\tau \geq$-uniformly convergent to $f$, there exists $\lambda_{0} \in \Lambda$ such that $f_{\lambda}(x) \leq f(x)$ for all $\lambda \geq \lambda_{0}$ and all $x \in X$. Obviously, this implies that $\left[f_{\lambda}\right]^{\alpha} \subseteq B_{d}\left([f]^{\alpha}, \varepsilon\right)$ for all $\lambda \geq \lambda_{0}, \varepsilon>0$ and $\alpha \in(0,1]$. 
$(2) \Rightarrow(3)$ By Proposition 4 we know that $\left\{f_{\lambda}\right\}_{\lambda \in \Lambda}$ is $\tau_{\geq}$-uniformly convergent to $M_{f}\left(B_{d}(\cdot, \varepsilon)\right)$ for all $\varepsilon>0$. Since $f$ is uniformly upper semicontinuous we can find $\delta_{\varepsilon}>0$ such that

$$
f(y)<f(x)+\varepsilon
$$

whenever $d(x, y)<\delta_{\varepsilon}$. Therefore, given $x \in X$ we have that

$$
M_{f}\left(B_{d}\left(x, \delta_{\varepsilon}\right)\right)<f(x)+\varepsilon .
$$

Furthermore, there exists $\lambda_{\delta_{\varepsilon}} \in \Lambda$ such that, $f_{\lambda}(x) \leq M_{f}\left(B_{d}\left(x, \delta_{\varepsilon}\right)\right)<f(x)+\varepsilon$ for all $\lambda \geq \lambda_{\delta_{\varepsilon}}$ and all $x \in X$. Consequently, $\left\{f_{\lambda}\right\}_{\lambda \in \Lambda}$ is upper uniformly convergent to $f$.

$(3) \Rightarrow(4)$ If $\left\{f_{\lambda}\right\}_{\lambda \in \Lambda}$ is upper uniformly convergent to $f$, given $\delta>0$ we can find $\lambda_{\delta} \in \Lambda$ such that $f_{\lambda}(x) \leq f(x)+\delta$ for all $\lambda \geq \lambda_{\delta}$ and all $x \in X$. Hence, given $\varepsilon>0$, then $M_{f_{\lambda}}\left(B_{d}(x, \varepsilon)\right) \leq M_{f}\left(B_{d}(x, \varepsilon)\right)+\delta$ for all $\lambda \geq \lambda_{\delta}$, so $\lim \sup _{\lambda \in \Lambda} M_{f_{\lambda}}\left(B_{d}(x, \varepsilon)\right) \leq M_{f}\left(B_{d}(x, \varepsilon)\right)+\delta$. Since $\delta$ is arbitrary we deduce that $\lim \sup _{\lambda \in \Lambda} M_{f_{\lambda}}\left(B_{d}(x, \varepsilon)\right) \leq M_{f}\left(B_{d}(x, \varepsilon)\right)$, so

$$
\inf _{\varepsilon>0} \limsup _{\lambda \in \Lambda} M_{f_{\lambda}}\left(B_{d}(x, \varepsilon)\right) \leq \inf _{\varepsilon>0} M_{f}\left(B_{d}(x, \varepsilon)\right)=\limsup _{x^{\prime} \rightarrow x} f\left(x^{\prime}\right)=f(x) .
$$

Therefore, $\left\{f_{\lambda}\right\}_{\lambda \in \Lambda}$ is $\Gamma^{+}$-convergent to $f$.

Next, we provide some examples showing that the converses of the implications of the above proposition are not true in general. Again, we suppose that $\mathbb{R}$ and all its subsets are endowed with the Euclidean metric $d_{e}$.

Example $9((2) \nRightarrow(1))$. Consider the sequence $\left\{f_{n}\right\}_{n \in \mathbb{N}}$ of upper semicontinuous fuzzy sets on I given by

$$
f_{n}(x)= \begin{cases}x+\frac{1}{n} & \text { if } \frac{1}{3 n} \leq x \leq 1-\frac{1}{3 n} \\ 0 & \text { otherwise }\end{cases}
$$

for all $n \in \mathbb{N}$. Let $f$ be the identity fuzzy set on $I$. Given $\varepsilon>0$, take $n_{\varepsilon} \in \mathbb{N}$ with $1 / n_{\varepsilon}<\varepsilon$. Let $\alpha \in(0,1], n \geq n_{\varepsilon}$ and $x \in\left[f_{n}\right]^{\alpha}, i . \quad$ e. $\alpha \leq f_{n}(x)=x+1 / n=$ $f(x+1 / n)$. Since $x+1 / n \in B_{d_{e}}(x, \varepsilon)$ then $x \in B_{d_{e}}\left([f]^{\alpha}, \varepsilon\right)$. Consequently, $\left\{f_{n}\right\}_{n \in \mathbb{N}}$ is $d_{\infty}^{+}$-convergent to $f$.

On the other hand, it is clear that $\left\{f_{n}\right\}_{n \in \mathbb{N}}$ is not $\tau_{\geq}$-uniformly convergent to $f$ since $f(x)<f_{n}(x)$ for all $x \in\left[\frac{1}{3}, \frac{2}{3}\right]$ and all $n \in \mathbb{N}$.

Example $10((3) \nRightarrow(2))$. Consider the fuzzy sets $f_{n}(x)=1 / 2+1 /(n+1)$ for all $n \in \mathbb{N}$ and all $x \in \mathbb{R}$. It is clear that the sequence $\left\{f_{n}\right\}_{n \in \mathbb{N}}$ is (upper-) uniformly convergent to the fuzzy set $f(x)=1 / 2$ for all $x \in \mathbb{R}$. Nevertheless, given $\varepsilon>0$ and $n \in \mathbb{N}$ we can find $\alpha \in(0,1]$ such that $1 / 2<\alpha<1 / 2+1 /(n+1)$ so $\left[f_{n}\right]^{\alpha}=\mathbb{R} \nsubseteq B_{d_{e}}\left([f]^{\alpha}, \varepsilon\right)=\varnothing$. Consequently, $\left\{f_{n}\right\}_{n \in \mathbb{N}}$ is not $d_{\infty}^{+}$-convergent to $f$.

Example $11((4) \nRightarrow(3))$. For each $n \in \mathbb{N}$, define $f_{n}: I \rightarrow I$ given by

$$
f_{n}(x)=\left\{\begin{array}{ll}
\frac{1}{2} & \text { if } x \geq \frac{1}{n} \\
1 & \text { otherwise }
\end{array} .\right.
$$

Since $\inf _{\varepsilon>0} \lim \sup _{n \in \mathbb{N}} M_{f_{n}}\left(B_{d}(x, \varepsilon)\right)=1 / 2$ for all $x \in I$, we deduce that $\left\{f_{n}\right\}_{n \in \mathbb{N}}$ is $\Gamma^{+}$-convergent to the identically $1 / 2$ fuzzy set $f$.

On the other hand, given $0<\varepsilon<1 / 2$ and $n \in \mathbb{N}$ then

$$
f_{n}(1 /(n+1))-f(1 /(n+1))=1 / 2>\varepsilon,
$$

so $\left\{f_{n}\right\}_{n \in \mathbb{N}}$ is not upper uniformly convergent to $f$. 
We notice that, in general, $d_{\infty}^{+}$-convergence does not imply $\Gamma^{+}$-convergence. In fact, $\Gamma^{+}$-convergence is not topological in general. For example, the constant sequence of fuzzy sets defined over $\mathbb{R}$ whose elements are the characteristic function of $\mathbb{R}-\{0\}$ is not $\Gamma^{+}$-convergent to itself. Observe that these fuzzy sets are not upper semicontinuous.

However, as in the lower part, we can provide a purely set-theoretic characterization of this convergence. Let $X$ be a nonempty set and let $\mathcal{P}(X)$ be the family of all subsets of $X$. Recall that a net $\left\{A_{\lambda}\right\}_{\lambda \in \Lambda}$ in $\mathcal{P}(X)$ is upper convergent to a set $A \in \mathcal{P}(X)$ if

$$
\limsup _{\lambda \in \Lambda} A_{\lambda}:=\bigcap_{\lambda_{0} \in \Lambda}\left(\bigcup_{\lambda \geq \lambda_{0}} A_{\lambda}\right) \subseteq A .
$$

Notice that if $(X, \tau)$ is a topological space then $\lim \sup _{\lambda \in \Lambda} A_{\lambda} \subseteq \operatorname{Ls} A_{\lambda}$. In contraposition with the lower part, $\lim \sup _{\lambda \in \Lambda} A_{\lambda}$ and $\operatorname{Ls} A_{\lambda}$ can be different even for discrete topological spaces.

This allows us to define the following uniform convergence for multifunctions:

Definition 11. Let $X$ be a nonempty set and let $\left\{f_{\lambda}\right\}_{\lambda \in \Lambda}$ be a net of multifunctions $f_{\lambda}: X \rightrightarrows I$. We say that the net $\left\{f_{\lambda}\right\}_{\lambda \in \Lambda}$ is upper uniformly convergent to the multifunction $f: X \rightrightarrows I$ if we can find $\lambda_{0} \in \Lambda$ such that

$$
\bigcup_{\lambda \geq \lambda_{0}} f_{\lambda}(x) \subseteq f(x) \text { for all } x \in X .
$$

The following result characterizes $d_{\infty}^{+}$-convergence in the most general context, where we use the fact that a fuzzy set $f$ on a metric space $(X, d)$ can be considered as a multifunction $f^{\prime}: X \rightrightarrows I$ such that $f^{\prime}(x)=\{f(x)\}$. In fact, we will not distinguish between $f$ and $f^{\prime}$.

Theorem 4. Let $(X, d)$ be a metric space. Then a net $\left\{f_{\lambda}\right\}_{\lambda \in \Lambda}$ in $\mathcal{F}(X)$ is $d_{\infty}^{+}$convergent to $f \in \mathcal{F}(X)$ if and only if the net $\left\{f_{\lambda}\right\}_{\lambda \in \Lambda}$ considered as a net of multifunctions is upper uniformly convergent to the multifunction $f^{\varepsilon}: X \rightrightarrows I$ for all $\varepsilon>0$ where $f^{\varepsilon}(x)={\overline{f\left(B_{d}(x, \varepsilon)\right)}}^{\tau}$.

Proof. Suppose that $\left\{f_{\lambda}\right\}_{\lambda \in \Lambda}$ is $d_{\infty}^{+}$-convergent to $f$. Given $\varepsilon>0$ we can find $\lambda_{\varepsilon} \in \Lambda$ such that $\left[f_{\lambda}\right]^{\alpha} \subseteq B_{d}\left([f]^{\alpha}, \varepsilon\right)$ for all $\lambda \geq \lambda_{\varepsilon}$ and all $\alpha \in(0,1]$. Fix $x \in X$ and $\lambda \geq \lambda_{\varepsilon}$. If $f_{\lambda}(x)=0$ then it is obvious that $f_{\lambda}(x) \in f^{\varepsilon}(x)=\downarrow f\left(B_{d}(x, \varepsilon)\right)$. If $f_{\lambda}(x) \neq 0$ then $\left[f_{\lambda}\right]^{f_{\lambda}(x)} \subseteq B_{d}\left([f]^{f_{\lambda}(x)}, \varepsilon\right)$ so we deduce that $f_{\lambda}(x) \in f^{\varepsilon}(x)$ which finishes this implication.

Now suppose that $\left\{f_{\lambda}\right\}_{\lambda \in \Lambda}$ is upper uniformly convergent to $f^{\varepsilon}$ for all $\varepsilon>0$. Then, given $\varepsilon>0$, there exists $\lambda_{\varepsilon} \in \Lambda$ such that $f_{\lambda}(x) \in f^{\varepsilon}(x)$ for all $\lambda \geq \lambda_{\varepsilon}$. Let $\alpha \in(0,1], \lambda \geq \lambda_{\varepsilon}$ and $x \in\left[f_{\lambda}\right]^{\alpha}$. Since $f_{\lambda}(x) \in f^{\varepsilon}(x)$ we can find $x_{\lambda} \in B_{d}(x, \varepsilon)$ such that $f_{\lambda}(x) \leq f\left(x_{\lambda}\right)$. Therefore $x \in B_{d}\left([f]^{\alpha}, \varepsilon\right)$. This concludes the proof.

Corollary 5. Let $(X, d)$ be a metric space. If the net $\left\{f_{\lambda}\right\}_{\lambda \in \Lambda}$ in $\mathcal{F}(X)$ is $d_{\infty}^{+-}$ convergent to $f \in \mathcal{F}(X)$ then

$$
\limsup _{\lambda \in \Lambda} \text { end } f_{\lambda} \subseteq \text { end } \bar{f} \text {. }
$$

Proof. If $\left\{f_{\lambda}\right\}_{\lambda \in \Lambda}$ is $d_{\infty}^{+}$-convergent to $f$, by the above theorem $\left\{f_{\lambda}\right\}_{\lambda \in \Lambda}$ is upper uniformly convergent to $f^{\varepsilon}$ for all $\varepsilon>0$. Therefore, given $\varepsilon>0$ we can find 
$\lambda_{\varepsilon} \in \Lambda$ such that $f_{\lambda}(x) \leq M_{f}\left(B_{d}(x, \varepsilon)\right)$ for all $\lambda \geq \lambda_{\varepsilon}$ and all $x \in X$. Hence, $\cup_{\lambda \geq \lambda_{\varepsilon}}$ end $f_{\lambda} \subseteq$ end $M_{f}\left(B_{d}(\cdot, \varepsilon)\right)$ so

$$
\limsup _{\lambda \in \Lambda} \text { end } f_{\lambda} \subseteq \bigcap_{\varepsilon>0} \bigcup_{\lambda \geq \lambda_{\varepsilon}} \text { end } f_{\lambda} \subseteq \bigcap_{\varepsilon>0} \text { end } M_{f}\left(B_{d}(\cdot, \varepsilon)\right)=\operatorname{end} \bar{f} \text {. }
$$

Observe that the converse of the previous result is not true in general (see Example 10).

Remark 17. Notice that when we consider upper semicontinuous fuzzy sets, the above result can be improved since $\left\{f_{\lambda}\right\}_{\lambda \in \Lambda}$ is $\Gamma^{+}$-convergent to $f$ so

$$
\text { Ls end } f_{\lambda} \subseteq \text { end } f \text {. }
$$

Corollary 6 (cf. [14, Lemma 3.3]). Let $(X, d)$ be a metric space. Then a net $\left\{f_{\lambda}\right\}_{\lambda \in \Lambda}$ in $S U S C(X)$ is $d_{\infty}^{+}$-convergent to $f \in S U S C(X)$ if and only if $\left\{f_{\lambda}\right\}_{\lambda \in \Lambda}$ is $\tau_{\geq}$-uniformly convergent to $M_{f}\left(B_{d}(\cdot, \varepsilon)\right)$ for all $\varepsilon>0$.

Proof. By Proposition 4, we only have to prove the sufficiency.

Let $\varepsilon>0$. By assumption, we can find $\lambda_{\varepsilon}$ such that $f_{\lambda}(x) \leq M_{f}\left(B_{d}(x, \varepsilon / 2)\right)$ for all $\lambda \geq \lambda_{\varepsilon}$ and all $x \in X$. We show that $\left[f_{\lambda}\right]^{\alpha} \subseteq B_{d}\left([f]^{\alpha}, \varepsilon\right)$ for all $\lambda \geq \lambda_{\varepsilon}$ and all $\alpha \in(0,1]$. Fix $\lambda \geq \lambda_{\varepsilon}$ and $\alpha \in(0,1]$. Let $x \in\left[f_{\lambda}\right]^{\alpha}$. If $\alpha \leq f_{\lambda}(x)<$ $M_{f}\left(B_{d}(x, \varepsilon / 2)\right)$ then it is obvious that $x \in B_{d}\left([f]^{\alpha}, \varepsilon\right)$. Otherwise, suppose that $f_{\lambda}(x)=M_{f}\left(B_{d}(x, \varepsilon / 2)\right)>0$ (if $f_{\lambda}(x)=0$ the conclusion is obvious). Then we can find a sequence $\left\{a_{n}\right\}_{n \in \mathbb{N}}$ in $B_{d}(x, \varepsilon / 2)$ such that $f\left(a_{n}\right)>0$ for all $n \in \mathbb{N}$ and $\lim _{n \rightarrow+\infty} f\left(a_{n}\right)=f_{\lambda}(x)$. Since $[f]^{0}$ is compact, we can suppose without loss of generality that the sequence $\left\{a_{n}\right\}_{n \in \mathbb{N}}$ is convergent to an $a \in \overline{B_{d}}(x, \varepsilon / 2)$. Since $f$ is upper semicontinuous we have that $\limsup _{n \rightarrow+\infty} f\left(a_{n}\right)=f_{\lambda}(x) \leq f(a)$. We conclude again that $x \in B_{d}\left([f]^{\alpha}, \varepsilon\right)$. Consequently, $\left\{f_{\lambda}\right\}_{\lambda \in \Lambda}$ is $d_{\infty}^{+}$-convergent to $f$.

Corollary 7. Let $(X, d)$ be a metric space. Then a net $\left\{f_{\lambda}\right\}_{\lambda \in \Lambda}$ in $\mathcal{F}(X)$ is $d_{\infty}^{+}$convergent to $f$ if and only if for all $\varepsilon>0$ there exists $\lambda_{\varepsilon} \in \Lambda$ such that

$$
f_{\lambda}(x) \leq M_{f}\left(B_{d}(x, \varepsilon)\right) \text { for all } \lambda \geq \lambda_{\varepsilon} \text { and all } x \in X
$$

and

$$
\text { if given } \lambda \geq \lambda_{\varepsilon}, f_{\lambda}(x)=M_{f}\left(B_{d}(x, \varepsilon)\right) \text { then } \operatorname{Argmax}_{B_{d}(x, \varepsilon)} f \neq \varnothing \text {. }
$$

4.3. Convergence in the supremum metric. From the results obtained in the last two sections, it is easy to state results about the convergence in the supremum metric only combining the corresponding fact about the lower and upper parts. For instance:

Theorem 5. Let $(X, d)$ be a metric space. Then a net $\left\{f_{\lambda}\right\}_{\lambda \in \Lambda}$ in $\mathcal{F}(X)$ is $d_{\infty}$ convergent to $f \in \mathcal{F}(X)$ if and only if:

(1) the net of multifunctions $\left\{f_{\lambda}^{\varepsilon}\right\}_{\lambda \in \Lambda}$ is lower uniformly convergent to $f$ for all $\varepsilon>0$, where $f_{\lambda}^{\varepsilon}: X \rightrightarrows I$ is given by $f_{\lambda}^{\varepsilon}(x)={\overline{f_{\lambda}\left(B_{d}(x, \varepsilon)\right.}}^{\tau}{ }^{\tau}$;

(2) the net $\left\{f_{\lambda}\right\}_{\lambda \in \Lambda}$ considered as a net of multifunctions is upper uniformly convergent to the multifunction $f^{\varepsilon}: X \rightrightarrows I$ for all $\varepsilon>0$ where $f^{\varepsilon}(x)=$ ${\overline{f\left(B_{d}(x, \varepsilon)\right)^{\tau}}}^{\tau}$.

Corollary 8 ([14, Lemma 3.3]). Let $(X, d)$ be a metric space. Then a net $\left\{f_{\lambda}\right\}_{\lambda \in \Lambda}$ in $S U S C(X)$ is $d_{\infty}$-convergent to $f \in S U S C(X)$ if and only if: 
(1) $\left\{M_{f_{\lambda}}\left(B_{d}(\cdot, \varepsilon)\right)\right\}_{\lambda \in \Lambda}$ is $\tau_{\leq}$-uniformly convergent to $f$ for all $\varepsilon>0$;

(2) $\left\{f_{\lambda}\right\}_{\lambda \in \Lambda}$ is $\tau_{\geq}$-uniformly convergent to $M_{f}\left(B_{d}(\cdot, \varepsilon)\right)$ for all $\varepsilon>0$.

Finally we will treat explicitly some questions about maximization when considering the $d_{\infty}$-convergence.

Proposition 6. Let $(X, d)$ be a metric space. If a net $\left\{f_{\lambda}\right\}_{\lambda \in \Lambda}$ in $\mathcal{F}(X)$ is $d_{\infty}$ convergent to $f$ then $\left\{M_{f_{\lambda}}(X)\right\}_{\lambda \in \Lambda}$ is residually constant and equal to $M_{f}(X)$.

Proof. This is a consequence of Remarks 11 and 15.

Proposition 7. Let $(X, d)$ be a metric space and let $\left\{f_{\lambda}\right\}_{\lambda \in \Lambda}$ be a net in $\mathcal{F}(X)$ $d_{\infty}$-convergent to an upper semicontinuous fuzzy set $f$. If $x_{\lambda} \in \operatorname{Argmax}_{X} f_{\lambda}$ for all $\lambda \in \Lambda$ and $\left\{x_{\lambda}\right\}_{\lambda \in \Lambda}$ is convergent to $x_{0}$ then $x_{0} \in \operatorname{Argmax}_{X} f$.

Proof. Suppose that $x_{\lambda} \in \operatorname{Argmax}_{X} f_{\lambda}$ for all $\lambda \in \Lambda$ and that $\left\{x_{\lambda}\right\}_{\lambda \in \Lambda}$ is convergent to $x_{0}$. Since $\left\{f_{\lambda}\right\}_{\lambda \in \Lambda}$ is $d_{\infty}$-convergent to $f$, by Proposition 4 given $\varepsilon>0$ we can find $\lambda_{\varepsilon} \in \Lambda$ such that

$$
f_{\lambda}(x) \leq M_{f}\left(B_{d}\left(x_{\lambda}, \varepsilon / 2\right)\right) \text { for all } x \in X, \lambda \geq \lambda_{\varepsilon},
$$

and

$$
d\left(x_{0}, x_{\lambda}\right)<\varepsilon / 2 \text { for all } \lambda \geq \lambda_{\varepsilon} .
$$

In particular, and since $B_{d}\left(x_{\lambda}, \varepsilon / 2\right) \subseteq B_{d}\left(x_{0}, \varepsilon\right)$ for all $\lambda \geq \lambda_{\varepsilon}$, we have that

$$
f_{\lambda}\left(x_{\lambda}\right)=M_{f_{\lambda}}(X) \leq M_{f}\left(B_{d}\left(x_{\lambda}, \varepsilon / 2\right)\right) \leq M_{f}\left(B_{d}\left(x_{0}, \varepsilon\right)\right) \leq M_{f}(X)
$$

for all $\lambda \geq \lambda_{\varepsilon}$. By the above proposition we know that $M_{f}(X)=M_{f_{\lambda}}(X)$ residually so

$$
M_{f_{\lambda}}(X)=M_{f}\left(B_{d}\left(x_{0}, \varepsilon\right)\right)=M_{f}(X) \text { residually. }
$$

Since this is valid for all $\varepsilon>0$ and $f$ is upper semicontinuous then $\lim _{\sup _{x \rightarrow x_{0}}} f(x)=$ $\inf _{\varepsilon>0} M_{f}\left(B_{d}\left(x_{0}, \varepsilon\right)\right)=f\left(x_{0}\right)=M_{f}(X)$.

Remark 18. Notice that the previous proposition is not true if we don't consider upper semicontinuous fuzzy sets. For example, consider the net of fuzzy sets $\left\{f_{\lambda}\right\}_{\lambda \in \Lambda}$ on $I$ which are equally constant to 1 and the fuzzy set in I given by

$$
f(x)=\left\{\begin{array}{ll}
1 & \text { if } 0<x \leq 1 \\
1 / 2 & \text { if } x=0
\end{array} .\right.
$$

It is obvious that $\left\{f_{\lambda}\right\}_{\lambda \in \Lambda}$ is $d_{\infty}$-convergent to $f$. Nevertheless, $0 \in \operatorname{Argmax}_{X} f_{\lambda}$ for all $\lambda \in \Lambda$ but $0 \notin \operatorname{Argmax}_{X} f$.

\section{ACKNOWLEDGEMENTS}

The authors are grateful to the referee for his/her valuable comments which have improved the paper.

\section{REFERENCES}

[1] R. Beattie and H.-P. Butzmann, Convergence Structures and Applications to Functional Analysis, Kluwer Academic Publishers, 2002.

[2] G. Beer, Topologies on Closed and Closed Convex Sets, vol. 268, Kluwer Academic Publishers, 1993.

[3] G. Beer and P. Kenderov, Epiconvergence and Baire category, Boll. Un. Mat. Ital. B (7) 3, no. 1 (1989), 41-56.

[4] G. Beer and P. Kenderov, On the arg min multifunction for lower semicontinuous functions, Proc. Amer. Math. Soc. 102, no. 1 (1988), 107-113. 
[5] G. Beer and R. Lucchetti, Convex optimization and the epi-distance topology, Trans. Amer. Math. Soc. 327, no. 2 (1991), 795-813.

[6] G. Beer, R. T. Rockafella and R. Wets, A characterization of epi-convergence in terms of convergence of level sets, Proc. Amer. Math. Soc. 116, no. 3 (1992), 753-761.

[7] E. De Giorgi and T. Franzoni, Su un tipo di convergenza variazionale, Atti Accad. Naz. Lincei, VIII. Ser., Rend., Cl. Sci. Fis. Mat. Nat. 58 (1975), 842-850.

[8] S. Dolecki, Convergence of minima in convergence spaces, Optimization 17, no. 5 (1986), $553-572$.

[9] S. Dolecki, G. Salinetti, and R. J.-B. Wets, Convergence of functions: equi-semicontinuity, Trans. Amer. Math. Soc. 276 (1983), 409-430.

[10] J.-X. Fang and Q.-Y. Xue, Some properties of the space of fuzzy-valued continuous functions on a compact set, Fuzzy Sets Syst. 160, no. 11 (2009), 1620-1631.

[11] G. Gierz, K. H. Hoffmann, K. Keimel, J. D. Lawson, M. Mislove, and D. S. Scott, A Compendium of Continuous Lattices, Springer-Verlag, 1980.

[12] G. H. Greco, A characterization of relatively compact sets of fuzzy sets, Nonlinear Anal.Theor., 64 no. 3 (2006), 518-529.

[13] G. H. Greco and M. P. Moschen, Supremum metric and relatively compact sets of fuzzy sets, Nonlinear Anal.-Theor. 64, no. 6 (2006), 1325-1335.

[14] G. H. Greco, M. P. Moschen, and E. Quelho Frota Rezende, On the variational convergence of fuzzy sets in metric spaces, Ann. Univ. Ferrara, Nuova Ser., Sez. VII 44 (1998), 27-39.

[15] V. Gregori and S. Romaguera, Approximate quantities, hyperspaces and metric completeness, Boll. Unione Mat. Italiana (8) 3-B (2000), 751-755.

[16] S. Heilpern, Fuzzy mappings and fixed point theorem, J. Math. Anal. Appl. 83 (1981), 566569.

[17] O. Kaleva, On the convergence of fuzzy sets, Fuzzy Sets Syst. 17 (1985), 53-65.

[18] O. Kaleva, A characterization of level-continuous fuzzy numbers, Fuzzy Sets Syst. 209 (2012), $84-88$.

[19] J. L. Kelley, General Topology, Springer, 1955.

[20] P. E. Kloeden, Compact supported endographs and fuzzy sets, Fuzzy Sets Syst. 4 (1980), 193-201.

[21] R. Lucchetti, Convexity and Well-Posed Problems, CMS Books in Mathematics, Springer, 2006.

[22] M. L. Puri and D. A. Ralescu, Differentials of fuzzy functions, J. Math. Anal. Appl. 91 (1983), 552-558.

[23] D. Qiu and L. Shu, Supremum metric on the space of fuzzy sets and common fixed point theorems for fuzzy mappings, Inf. Sci. 178, no. 18 (2008), 3595-3604.

[24] R. T. Rockafellar and R. J.-B. Wets, Variational Analysis, Comprehensive Studies in Mathematics, vol. 317, Springer, 1998.

[25] M. Rojas-Medar and H. Román-Flores, On the equivalence of convergences of fuzzy sets, Fuzzy Sets Syst. 80, no. 2 (1996), 217-224.

[26] W. Trutschnig, Characterization of the sendograph-convergence of fuzzy sets by means of their $L_{p^{-}}$and levelwise convergence, Fuzzy Sets Syst. 161, no. 8 (2010), 1064-1077.

[27] R. A. Wijsman, Convergence of sequences of convex sets, cones, and functions, Bull. Amer. Math. Soc. 70 (1964), 186-188.

(Tatiana Pedraza) Instituto Universitario de Matemática Pura y Aplicada, Universitat Politècnica de ValÈnCia, 46022 Valencia, Spain.

E-mail address: tapedraz@mat.upv.es

(Jesús Rodríguez-López) Instituto Universitario de Matemática Pura y Aplicada, Universitat Politècnica de València, 46022 Valencia, Spain.

E-mail address: jrlopez@mat.upv.es

(Salvador Romaguera) Instituto Universitario de Matemática Pura y Aplicada, Universitat Politècnica de València, 46022 Valencia, Spain.

E-mail address: sromague@mat.upv.es 